\title{
Wavelet transforms on Gelfand-Shilov spaces and concrete examples
}

\author{
Naohiro Fukuda', Tamotu Kinoshita ${ }^{2 *}$ and Kazuhisa Yoshino ${ }^{2}$
}

\author{
"Correspondence: \\ kinosita@math.tsukuba.ac.jp \\ ${ }^{2}$ Institute of Mathematics, Tsukuba \\ University, Tsukuba, Ibaraki \\ 305-8571, Japan \\ Full list of author information is \\ available at the end of the article
}

\begin{abstract}
In this paper, we study the continuity properties of wavelet transforms in the Gelfand-Shilov spaces with the use of a vanishing moment condition. Moreover, we also compute the Fourier transforms and the wavelet transforms of concrete functions in the Gelfand-Shilov spaces.
\end{abstract}

MSC: 42C 10;46F05; 65T60

Keywords: wavelet transform; Gelfand-Shilov space; continuity properties

\section{Introduction}

In recent years, the wavelet transform has been shown to be a successful tool in signal processing applications such as data compression and fast computations. The wavelet transform of $f \in L^{2}(\mathbf{R})$ with respect to the analyzed wavelet $\psi \in L^{2}(\mathbf{R})$ satisfying the admissible condition $C_{\psi}:=\int_{\mathbf{R}}|\hat{\psi}(\xi)|^{2} /|\xi| d \xi<\infty$ is defined by

$$
W_{\psi} f(a, b)=\frac{1}{\sqrt{C_{\psi}}} \int_{\mathbf{R}} f(x) \overline{\psi_{a, b}(x)} d x,
$$

where

$$
\psi_{a, b}(x)=\frac{1}{\sqrt{a}} \psi\left(\frac{x-b}{a}\right) \quad(a>0, b \in \mathbf{R})
$$

(see [1, 2] for example). The inverse wavelet transform of $F \in L^{2}\left(\mathbf{R}_{+} \times \mathbf{R}\right)$ with respect to the analyzed wavelet $\psi \in L^{2}(\mathbf{R})$ is defined by

$$
M_{\psi} F(x)=\frac{1}{\sqrt{C_{\psi}}} \int_{\mathbf{R}_{+}} \int_{\mathbf{R}} F(a, b) \psi_{a, b}(x) \frac{d b d a}{a^{2}} \quad(x \in \mathbf{R}) .
$$

For the time-frequency analysis, we are concerned with better localization in both time and frequency spaces from a point of view of the uncertainty principle. For the wellbalanced localization, it would be suitable to consider the Schwartz space $\mathcal{S}$ regarded as the space of functions which have arbitrary polynomial decay and whose Fourier transforms also have arbitrary polynomial decay (see [3]). For instance, the typical Mexican hat wavelet belongs to spaces of more rapidly decreasing and more regular functions in $\mathcal{S}$. In

(c) The Author(s) 2017. This article is distributed under the terms of the Creative Commons Attribution 4.0 International License (http://creativecommons.org/licenses/by/4.0/), which permits unrestricted use, distribution, and reproduction in any medium, provided you give appropriate credit to the original author(s) and the source, provide a link to the Creative Commons license, and indicate if changes were made. 
this article we focus on Gelfand-Shilov spaces of functions which have sub-exponential decay and whose Fourier transforms also have sub-exponential decay. For positive constants $\mu, v$ and $h$ such that $\mu+v \geq 1$, we define the Banach Gelfand-Shilov space

$$
\mathcal{S}_{v, h}^{\mu}(\mathbf{R})=\left\{f \in \mathcal{S} ;\left\|x^{\alpha} \partial_{x}^{\beta} f(x)\right\|_{L^{\infty}(\mathbf{R})} \leq C h^{\alpha+\beta} \alpha !^{\nu} \beta !^{\mu} \text { for all } \alpha, \beta \in \mathbf{N}\right\}
$$

with the norm

$$
\|f\|_{\mathcal{S}_{v, h}^{\mu}(\mathbf{R})}=\sup _{\alpha, \beta \in \mathbf{N}} \frac{\left\|x^{\alpha} \partial_{x}^{\beta} f(x)\right\|_{L^{\infty}(\mathbf{R})}}{h^{\alpha+\beta} \alpha !^{\nu} \beta !^{\mu}}
$$

and the (non-Banach) Gelfand-Shilov space $\mathcal{S}_{v}^{\mu}(\mathbf{R})$

$$
\mathcal{S}_{v}^{\mu}(\mathbf{R})=\text { ind } \lim _{h>0} \mathcal{S}_{v, h}^{\mu}(\mathbf{R})
$$

with the inductive limit topology. The Gelfand-Shilov spaces were originally introduced in [4] and [5]. As well explained in [6] and [7], the Gelfand-Shilov spaces are better adapted to the study of the problems of partial differential equations for which the solutions subexponentially decay at infinity.

Remark 1.1 Restricting functions with Fourier transforms supported in the right halfplane, we may also define the Banach progressive Gelfand-Shilov space

$$
\mathcal{S}_{v, h}^{\mu,+}(\mathbf{R})=\left\{f \in \mathcal{S}_{v, h}^{\mu}(\mathbf{R}) ; \operatorname{supp} \hat{f} \subset[0, \infty)\right\},
$$

and the (non-Banach) progressive Gelfand-Shilov space

$$
\mathcal{S}_{v}^{\mu,+}(\mathbf{R})=\left\{f \in \mathcal{S}_{v}^{\mu}(\mathbf{R}) ; \operatorname{supp} \hat{f} \subset[0, \infty)\right\} .
$$

Such spaces can be considered in dealing with analytic signals as the Hardy space $H^{2}(\mathbf{R})$ (see [8]). If the analyzed wavelet $\psi$ belongs to the progressive Gelfand-Shilov space, $\hat{\psi}$ smoothly tends to zero and also has vanishing moments. For example, the Bessel wavelet $\psi(x)$ defined by $\hat{\psi}(\xi)=e^{-\xi-\xi^{-1}}$ for $\xi>0$ and $\hat{\psi}(\xi)=0$ for $\xi \leq 0$ belongs to $\mathcal{S}_{2}^{1,+}(\mathbf{R})$. Actually, we know that $\psi(x)=\frac{1}{\pi \sqrt{1-i x}} K_{1}(2 \sqrt{1-i x})$, where $K_{1}$ is the first modified Bessel function of the second kind (see [9]).

For the discrete wavelet case requiring strong additional conditions, the Meyer wavelets or the Gevrey wavelets constructed as in [10] belong to the Gelfand-Shilov spaces. As for the continuous wavelet transform requiring only the admissible condition, there are many possibilities to choose the analyzed wavelet. Boundedness results in a generalized Sobolev space, Besov space and Lizorkin-Triebel space are given in [3]. As for $\psi \in \mathcal{S}_{v}^{\mu}(\mathbf{R})$ and $\psi \in$ $\mathcal{S}_{v}^{\mu,+}(\mathbf{R}),[7]$ and [11] show the continuity properties of wavelet transforms by preparing spaces of functions in $a$ and $b$, respectively. In this paper, we shall pay careful attention also to the parameter $h$ as the radius of convergence in the analytic class and attempt to find a further detailed estimate with $h$. So, our purpose is to show the continuity properties in (strong) topologies of Banach Gelfand-Shilov spaces with the use of a vanishing moment condition and to give concrete examples which can indicate the optimality in Section 4. 


\section{Results}

To state our results, we also introduce the following lemma.

Lemma 2.1 There exist $C>0$ and $h_{0}>0$ such that

$$
\left\|e^{h_{0}|x|^{1 / \nu}} f\right\|_{L^{\infty}(\mathbf{R})}+\left\|e^{h_{0}|\xi|^{1 / \mu}} \hat{f}\right\|_{L^{\infty}(\mathbf{R})} \leq C
$$

if and only iff $\in \mathcal{S}_{v, h}^{\mu}(\mathbf{R})$.

For the proof refer to $[6,12]$, etc. Taking Lemma 2.1 into account, we denote another Banach Gelfand-Shilov space combining with the infinite vanishing moment condition $|\hat{f}(\xi)| \leq C e^{-h|\xi|^{-1 / \delta}}$

$$
\left.S_{\nu, h}^{\mu, \delta}(\mathbf{R})=\left\{f \in \mathcal{S} ;\left\|e^{h|x|^{1 / v}} f\right\|_{L^{\infty}(\mathbf{R})}+\| e^{h \max \left\{|\xi|^{1 / \mu},|\xi|^{-1 / \delta}\right.}\right\} \hat{f} \|_{L^{\infty}(\mathbf{R})}<\infty\right\}
$$

We remark that $\mathcal{S}_{v, h}^{\mu}(\mathbf{R})$ corresponds to $S_{v, h}^{\mu, \delta}(\mathbf{R})$ with $\delta=\infty$, i.e.,

$$
S_{v, h}^{\mu, \infty}(\mathbf{R})=\left\{f \in \mathcal{S} ;\left\|e^{h|x|^{1 / v}} f\right\|_{L^{\infty}(\mathbf{R})}+\left\|e^{h|\xi|^{1 / \mu}} \hat{f}\right\|_{L^{\infty}(\mathbf{R})}<\infty\right\}
$$

Remark 2.2 In particular, when $\hat{f}(\xi)$ is just equal to $e^{-h|\xi|^{-1 / \delta}}$, it belongs to the Gevrey space of order $\delta+1$. So, $v$ can be taken as $v \geq \delta+1$.

Remark 2.3 We easily obtain $e^{h \max \left\{|\xi|^{1 / \mu},|\xi|^{-1 / \delta}\right\}} \leq e^{h\left(|\xi|^{1 / \mu}+|\xi|^{-1 / \delta}\right)}$. On the other hand, the weight can be estimated from below as

$$
e^{h \max \left\{|\xi|^{1 / \mu},|\xi|^{-1 / \delta}\right\}} \geq e^{h\left(|\xi|^{1 / \mu}+|\xi|^{-1 / \delta}-1\right)} \geq c e^{h\left(|\xi|^{1 / \mu}+|\xi|^{-1 / \delta}\right)}
$$

Therefore, we find that $\left\|e^{h\left(|\xi|^{1 / \mu}+|\xi|^{-1 / \delta}\right)} \hat{f}\right\|_{L^{\infty}(\mathbf{R})} \sim\left\|e^{h \max \left\{|\xi|^{1 / \mu},|\xi|^{-1 / \delta}\right\}} \hat{f}\right\|_{L^{\infty}(\mathbf{R})}$.

Then we prove the following.

Theorem 2.4 Let $\mu, v, h, h^{\prime}$ and $\delta$ be positive constants such that $\mu+v \geq 1, h^{\prime}<h$. Define that $d(\lambda)=\lambda(\lambda-1)^{-1+1 / \lambda}$. Then, for the wavelet transform $W_{\psi}$ with the wavelet $\psi \in S_{v, h}^{\mu, \delta}(\mathbf{R})$, the following estimates hold:

$$
\begin{aligned}
& \text { for } f \in S_{v, h}^{\infty, \infty}(\mathbf{R}) \\
& \text { (i) } \quad\left\|\frac{e^{h|b /(a+1)|^{1 / v}}}{a^{1 / 2}+1} W_{\psi} f\right\|_{L^{\infty}\left(\mathbf{R}_{+} \times \mathbf{R}\right)} \leq C\left\|e^{h|x|^{1 / v}} f\right\|_{L^{\infty}(\mathbf{R})} \quad \text { if } v>1 \text {, } \\
& (\mathrm{i})^{\prime} \quad\left\|e^{h^{\prime} 2^{1-1 / v}|b /(a+1)|^{1 / v}} W_{\psi} f\right\|_{L^{\infty}\left(\mathbf{R}_{+} \times \mathbf{R}\right)} \leq C\left\|e^{h|x|^{1 / v}} f\right\|_{L^{\infty}(\mathbf{R})} \quad \text { if } 0<v \leq 1 \text {, } \\
& \text { for } f \in S_{\infty, h}^{\mu, \infty}(\mathbf{R}) \\
& \text { (ii) }\left\|\frac{a^{1 / 2} e^{h d(\delta / \mu+1)^{1 / \mu} a^{-1 /(\mu+\delta)}}}{a+1} W_{\psi} f\right\|_{L^{\infty}\left(\mathbf{R}_{+} \times \mathbf{R}\right)} \leq C\left\|e^{h|\xi|^{1 / \mu}} \hat{f}\right\|_{L^{\infty}(\mathbf{R})} \quad \text { if } \mu>1 \text {, } \\
& \text { (ii) } \quad\left\|a^{-1 / 2} e^{h^{\prime} 2^{1-1 / \mu} d(\delta / \mu+1)^{1 / \mu} a^{-1 /(\mu+\delta)}} W_{\psi} f\right\|_{L^{\infty}\left(\mathbf{R}_{+} \times \mathbf{R}\right)} \leq C\left\|e^{h|\xi|^{1 / \mu}} \hat{f}\right\|_{L^{\infty}(\mathbf{R})} \\
& \text { if } 0<\mu \leq 1 \text {, }
\end{aligned}
$$




$$
\begin{aligned}
& \text { for } f \in S_{\infty, h}^{\mu, \delta}(\mathbf{R}) \\
& \begin{aligned}
\text { (iii) } \quad\left\|\frac{a^{1 / 2} e^{h d(\delta / \mu+1)^{1 / \mu}\left(\max \left\{a, a^{-1}\right\}\right)^{1 /(\mu+\delta)}}}{a+1} W_{\psi} f\right\|_{L^{\infty}\left(\mathbf{R}_{+} \times \mathbf{R}\right)} \\
\leq C\left\|e^{h \max \left\{|\xi|^{1 / \mu},|\xi|^{-1 / \delta}\right\}} \hat{f}\right\|_{L^{\infty}(\mathbf{R})} \quad \text { if } \mu>1, \\
\text { (iii) } \quad\left\|a^{-1 / 2} e^{h^{\prime} 2^{1-1 / \mu} d(\delta / \mu+1)^{1 / \mu}\left(\max \left\{a, a^{-1}\right\}\right)^{1 /(\mu+\delta)}} W_{\psi} f\right\|_{L^{\infty}\left(\mathbf{R}_{+} \times \mathbf{R}\right)} \\
\leq C\left\|e^{h \max \left\{|\xi|^{1 / \mu},|\xi|^{-1 / \delta}\right\}} \hat{f}\right\|_{L^{\infty}(\mathbf{R})} \quad \text { if } 0<\mu \leq 1 .
\end{aligned}
\end{aligned}
$$

Remark 2.5 We find that $d(\lambda)$ is strictly greater than 1 for $\lambda>1$ since $d^{\prime}(\lambda)=-(\lambda-$ $1)^{-1+1 / \lambda} \lambda^{-1} \log (\lambda-1)$, and $d(\lambda)$ has the maximum at the point $\lambda=2$ and $\lim _{\lambda \rightarrow 1+} d(\lambda)=$ $\lim _{\lambda \rightarrow \infty} d(\lambda)=1$.

Remark 2.6 This work is motivated by [7] where $f$ and $\psi$ are allowed to take each different value of parameters $v, \mu$ and have infinite vanishing moments, more precisely vanishing moments of arbitrary polynomial order. Therefore, we have restricted ourselves to the case of $f$ and $\psi$ under the common parameters $\nu, \mu$, and have derived the above estimates with $\delta$ (concerning vanishing moments of sub-exponential order). For instance, $\left\|e^{h\left(|b|^{1 /(2 v+\mu-1)}+\left(\max \left\{a, a^{-1}\right\}\right)^{1 /(v+\mu-1)}\right)} W_{\psi} f\right\|_{L^{\infty}}$ is estimated by [7] with $\rho_{1}=s=\mu$, $\rho_{2}=v, \tau_{1}=\tau_{2}=v+\mu-1$ and $t=2 v+\mu-1$ (in the case of $f$ and $\psi$ under the common parameters $v$ and $\mu$ ). If one considers small $a>0$ and takes $v=\delta+1$ (see $\operatorname{Re}$ mark 2.2), a similar estimate as (ii) holds since $\left(\max \left\{a, a^{-1}\right\}\right)^{1 /(v+\mu-1)}\left(\max \left\{a, a^{-1}\right\}\right)^{1 /(\mu+\delta)} \sim$ $a^{-1 /(\mu+\delta)}$. Thanks to the additional condition of sub-exponential order, (i) for small $a>0$ can become better since $\mu+v \geq 1$ and $|b|^{1 /(2 v+\mu-1)}=|b|^{1 /(v+(\mu+\nu-1))} \leq|b|^{1 / v} \sim \mid b /$ $\left.(a+1)\right|^{1 / v}$.

Considering the study of the continuity properties in $[3,7]$ and [11], we introduce spaces of functions in $a$ and $b$ which correspond to the Gelfand-Shilov spaces of functions in $x$ and $\xi$ since $a \sim 1 /|\xi|$ and $b \sim x$ after wavelet transforms. Therefore, we shall define the following weighted $L^{\infty}\left(\mathbf{R}_{+} \times \mathbf{R}\right)$ space which is a subspace of $L^{2}\left(\mathbf{R}_{+} \times \mathbf{R}\right)$ as far as $h$ is positive:

$$
V_{v, h}^{\mu, \delta}\left(\mathbf{R}_{+} \times \mathbf{R}\right)=\left\{F \in L^{2}\left(\mathbf{R}_{+} \times \mathbf{R}\right) ;\left\|e^{h \max \left\{|b /(a+1)|^{1 / v}, a^{1 / \mu}, a^{-1 / \delta}\right\}} F\right\|_{L^{\infty}\left(\mathbf{R}_{+} \times \mathbf{R}\right)}<\infty\right\} .
$$

We remark that if $\mu=\infty$,

$$
V_{v, h}^{\infty, \delta}\left(\mathbf{R}_{+} \times \mathbf{R}\right)=\left\{F \in L^{2}\left(\mathbf{R}_{+} \times \mathbf{R}\right) ;\left\|e^{h \max \left\{|b /(a+1)|^{1 / v}, a^{-1 / \delta}\right\}} F\right\|_{L^{\infty}\left(\mathbf{R}_{+} \times \mathbf{R}\right)}<\infty\right\} .
$$

By (i) and (ii), we have

$$
\begin{aligned}
& \left\|\left\{\frac{e^{h|b /(a+1)|^{1 / v}}}{a^{1 / 2}+1}+\frac{a^{1 / 2} e^{h d(\delta / \mu+1)^{1 / \mu} a^{-1 /(\mu+\delta)}}}{a+1}\right\} W_{\psi} f\right\|_{L^{\infty}\left(\mathbf{R}_{+} \times \mathbf{R}\right)} \\
& \leq C\left(\left\|e^{h|x|^{1 / v}} f\right\|_{L^{\infty}(\mathbf{R})}+\left\|e^{h|\xi|^{1 / \mu}} \hat{f}\right\|_{L^{\infty}(\mathbf{R})}\right) .
\end{aligned}
$$


The weight function can be estimated from below as

$$
\frac{e^{h|b /(a+1)|^{1 / v}}}{a^{1 / 2}+1}+\frac{a^{1 / 2} e^{h d(\delta / \mu+1)^{1 / \mu} a^{-1 /(\mu+\delta)}}}{a+1} \geq c e^{h \max \left\{|b /(a+1)|^{1 / v}, a^{-1 /(\mu+\delta)}\right\}}
$$

here we used Remark 2.5 also to eliminate the term $a^{1 / 2}$. Therefore, by Theorem 2.4, we can also get the following continuity properties.

Corollary 2.7 Let $\mu, v, h$ and $\delta$ be constants such that $\mu>1, v>1, h>0$ and $\delta>0$. Then, for the wavelet $\psi \in S_{v, h}^{\mu, \delta}(\mathbf{R})$, the wavelet transform $S_{v, h}^{\mu, \infty}(\mathbf{R}) \ni f \mapsto W_{\psi} f \in V_{v, h}^{\infty, \mu+\delta}\left(\mathbf{R}_{+} \times \mathbf{R}\right)$ is continuous. In particular, when $f$ also satisfies the infinite vanishing moment condition, the wavelet transform $S_{\infty, h}^{\mu, \delta}(\mathbf{R}) \ni f \mapsto W_{\psi} f \in V_{\infty, h}^{\mu+\delta, \mu+\delta}\left(\mathbf{R}_{+} \times \mathbf{R}\right)$ is continuous.

In Section 4 we shall discuss the optimality of our boundedness results in Gelfand-Shilov spaces.

\section{Proof of Theorem 2.4}

At first, we introduce the following lemma.

Lemma 3.1 It holds that for $\alpha, \beta \geq 0$

$$
\alpha^{1 / \theta}+\beta^{1 / \theta} \geq \begin{cases}2^{1-1 / \theta}(\alpha+\beta)^{1 / \theta} & \text { if } 0<\theta \leq 1 \\ (\alpha+\beta)^{1 / \theta}+\left(2-2^{1 / \theta}\right) \min \left\{\alpha^{1 / \theta}, \beta^{1 / \theta}\right\} & \text { if } \theta>1 .\end{cases}
$$

Remark 3.2 The latter inequality is given in [13] and [14], which also shows multiplication algebras for the Gevrey-modulation spaces.

Proof of Lemma 3.1 We shall suppose that $\alpha \geq \beta>0$ since the proof is trivial when $\alpha=0$ or $\beta=0$. Putting $\gamma:=\alpha / \beta(\geq 1)$, we may show

$$
\gamma^{1 / \theta}+1 \geq \begin{cases}2^{1-1 / \theta}(\gamma+1)^{1 / \theta} & \text { if } 0<\theta \leq 1 \\ (\gamma+1)^{1 / \theta}+2-2^{1 / \theta} & \text { if } \theta>1\end{cases}
$$

This follows from

$$
\min _{\gamma \geq 1}\left\{\frac{\gamma^{1 / \theta}+1}{(\gamma+1)^{1 / \theta}}\right\}=2^{1-1 / \theta} \quad \text { if } 0<\theta \leq 1
$$

and

$$
\min _{\gamma \geq 1}\left\{\gamma^{1 / \theta}+1-(\gamma+1)^{1 / \theta}\right\}=2-2^{1 / \theta} \quad \text { if } \theta>1 .
$$

In the proofs of theorems, $\|\cdot\|$ denotes the $L^{\infty}$ norm on $\mathbf{R}$ or $\mathbf{R}_{+} \times \mathbf{R}$. We shall consider the following cases.

- Case of $v>1$ and $a \geq 1$ ) From the definition of the wavelet transform we get

$$
\left|e^{h|b / \max \{1, a\}|^{1 / v}} W_{\psi} f(a, b)\right| \leq \frac{C\left\|e^{h|x|^{1 / v}} f\right\|}{a^{1 / 2}} \int_{\mathbf{R}} e^{-h\left\{|x|^{1 / v}+\left.|(x-b)| a\right|^{1 / v}-|b / \max \{1, a\}|^{1 / v}\right\}} d x .
$$


Lemma 3.1 with $\alpha=|b / a-x / a|, \beta=|x / a|$ gives

$$
\begin{aligned}
&|x|^{1 / v}+\left|\frac{x-b}{a}\right|^{1 / v}-\left|\frac{b}{\max \{1, a\}}\right|^{1 / v} \\
&=\left|\frac{b}{a}-\frac{x}{a}\right|^{1 / v}+\left|\frac{x}{a}\right|^{1 / v}-\left|\frac{b}{a}\right|^{1 / v}+|x|^{1 / v}\left\{1-\left|\frac{1}{a}\right|^{1 / v}\right\} \\
& \geq\left(\left|\frac{b}{a}-\frac{x}{a}\right|+\left|\frac{x}{a}\right|\right)^{1 / v}+\left(2-2^{1 / v}\right) \min \left\{\left|\frac{b}{a}-\frac{x}{a}\right|^{1 / v},\left|\frac{x}{a}\right|^{1 / v}\right\}-\left|\frac{b}{a}\right|^{1 / v} \\
&+|x|^{1 / v}\left\{1-\left|\frac{1}{a}\right|^{1 / v}\right\} \\
& \geq\left(2-2^{1 / v}\right) \min \left\{\left|\frac{b}{a}-\frac{x}{a}\right|^{1 / v},\left|\frac{x}{a}\right|^{1 / v}\right\}+|x|^{1 / v}\left\{1-\left|\frac{1}{a}\right|^{1 / v}\right\}
\end{aligned}
$$

here we used $(|b / a-x / a|+|x / a|)^{1 / v} \geq|b / a|^{1 / v}$. Therefore, putting $D:=\{x \in \mathbf{R} ;|b / a-x / a|<$ $|x / a|\}$, we have

$$
\begin{aligned}
\left|e^{h|b / \max \{1, a\}|^{1 / v}} W_{\psi} f(a, b)\right| \leq & \frac{C\left\|e^{h|x|^{1 / v}} f\right\|}{a^{1 / 2}} \int_{D} e^{-h\left(2-2^{1 / v}\right)|b / a-x / a|^{1 / v}} d x \\
& +\frac{C\left\|e^{h|x|^{1 / v}} f\right\|}{a^{1 / 2}} \int_{\mathbf{R} \backslash D} e^{-h\left(2-2^{1 / v}\right)|x / a|^{1 / v}} d x \\
\leq & 2 C a^{1 / 2}\left\|e^{h|x|^{1 / v}} f\right\| \int_{\mathbf{R}} e^{-h\left(2-2^{1 / v}\right)|x|^{1 / v}} d x \\
\leq & C a^{1 / 2}\left\|e^{h|x|^{1 / v}} f\right\| .
\end{aligned}
$$

- Case of $v>1$ and $0<a<1)$ Lemma 3.1 with $\alpha=|b-x|, \beta=|x|$ gives

$$
\begin{aligned}
& |x|^{1 / v}+\left|\frac{x-b}{a}\right|^{1 / v}-\left|\frac{b}{\max \{1, a\}}\right|^{1 / v} \\
& \quad=|b-x|^{1 / v}+|x|^{1 / v}-|b|^{1 / v}+|b-x|^{1 / v}\left\{\left|\frac{1}{a}\right|^{1 / v}-1\right\} \\
& \geq(|b-x|+|x|)^{1 / v}+\left(2-2^{1 / v}\right) \min \left\{|b-x|^{1 / v},|x|^{1 / v}\right\}-|b|^{1 / v}+|b-x|^{1 / v}\left\{\left|\frac{1}{a}\right|^{1 / v}-1\right\} \\
& \geq\left(2-2^{1 / v}\right) \min \left\{|b-x|^{1 / v},|x|^{1 / v}\right\}+|b-x|^{1 / v}\left\{\left|\frac{1}{a}\right|^{1 / v}-1\right\},
\end{aligned}
$$

here we used $(|b-x|+|x|)^{1 / \nu} \geq|b|^{1 / \nu}$. Therefore, putting

$$
I:=\{x \in \mathbf{R} ;|b-x|<1\}
$$

we find that

$$
\begin{aligned}
\int_{I} e^{-h|b-x|^{1 / v}\left\{|1 / a|^{1 / v}-1\right\}} d x & =\int_{b-1}^{b+1} e^{-h|(b-x) / a|^{1 / v}\left\{1-a^{1 / v}\right\}} d x \\
& =a \int_{-1 / a}^{1 / a} e^{-h|x|^{1 / v}\left\{1-a^{1 / v}\right\}} d x \leq M_{h, v} a
\end{aligned}
$$


for $0<a<1$ and have

$$
\begin{aligned}
& \left|e^{h|b / \max \{1, a\}|^{1 / v}} W_{\psi} f(a, b)\right| \\
& \leq \frac{C\left\|e^{h|x|^{1 / v}} f\right\|}{a^{1 / 2}} \int_{I} e^{-h|b-x|^{1 / v}\left\{|1 / a|^{1 / v}-1\right\}} d x \\
& \quad+\frac{C\left\|e^{h|x|^{1 / v}} f\right\|}{a^{1 / 2}} \int_{\mathbf{R} \backslash I} e^{-h\left\{\left(2-2^{1 / v}\right) \min \left\{|b-x|^{1 / v},|x|^{1 / v}\right\}+|1 / a|^{1 / v}-1\right\}} d x \\
& \leq M_{h, v} a^{1 / 2}\left\|e^{h|x|^{1 / v}} f\right\| \\
& \quad+M_{h, v}^{\prime}\left\|e^{h|x|^{1 / v}} f\right\| \int_{\mathbf{R}} e^{-h\left(2-2^{1 / v}\right) \min \left\{|b-x|^{1 / v},|x|^{1 / v}\right\}} d x \\
& \leq C\left\|e^{h|x|^{1 / v}} f\right\|,
\end{aligned}
$$

here we used $\frac{1}{a^{1 / 2}} e^{-h\left\{|1 / a|^{1 / v}-1\right\}} \leq M_{h, v}^{\prime}$ for $0<a<1$.

Thus, since $\max \{1, a\} \leq a+1$ and $\max \left\{1, a^{1 / 2}\right\} \sim a^{1 / 2}+1$, it follows that

$$
\left\|\frac{e^{h|b /(a+1)|^{1 / v}}}{a^{1 / 2}+1} W_{\psi} f\right\| \leq C\left\|e^{h|x|^{1 / v}} f\right\| .
$$

- Case of $0<v \leq 1$ and $a \geq 1$ ) For $h>h^{\prime}>0$, we get

$$
\begin{aligned}
& \left|e^{h^{\prime} 2^{1-1 / v}|b / \max \{1, a\}|^{1 / v}} W_{\psi} f(a, b)\right| \\
& \leq \frac{C\left\|e^{h|x|^{1 / v}} f\right\|}{a^{1 / 2}} \\
& \quad \times \int_{-\infty}^{\infty} e^{-\left(h-h^{\prime}\right)\left\{|x|^{1 / v}+|(x-b) / a|^{1 / v}\right\}-h^{\prime}\left\{|x|^{1 / v}+|(x-b) / a|^{1 / v}-2^{1-1 / v}|b / \max \{1, a\}|^{1 / v}\right\}} d x .
\end{aligned}
$$

Lemma 3.1 with $\alpha=|b / a-x / a|, \beta=|x / a|$ gives

$$
\begin{aligned}
& |x|^{1 / v}+\left|\frac{x-b}{a}\right|^{1 / v}-2^{1-1 / v}\left|\frac{b}{\max \{1, a\}}\right|^{1 / v} \\
& =\left|\frac{b}{a}-\frac{x}{a}\right|^{1 / v}+\left|\frac{x}{a}\right|^{1 / v}-2^{1-1 / v}\left|\frac{b}{a}\right|^{1 / v}+|x|^{1 / v}\left\{1-\left|\frac{1}{a}\right|^{1 / v}\right\} \\
& \quad \geq 2^{1-1 / v}\left(\left|\frac{b}{a}-\frac{x}{a}\right|+\left|\frac{x}{a}\right|\right)^{1 / v}-2^{1-1 / v}\left|\frac{b}{a}\right|^{1 / v}+|x|^{1 / v}\left\{1-\left|\frac{1}{a}\right|^{1 / v}\right\} \\
& \quad \geq|x|^{1 / v}\left\{1-\left|\frac{1}{a}\right|^{1 / v}\right\} .
\end{aligned}
$$

Therefore, we have

$$
\begin{aligned}
\left|e^{h^{\prime} 2^{1-1 / v}|b / \max \{1, a\}|^{1 / v}} W_{\psi} f(a, b)\right| & \leq \frac{C\left\|e^{h|x|^{1 / v}} f\right\|}{a^{1 / 2}} \int_{-\infty}^{\infty} e^{-\left(h-h^{\prime}\right)\left\{|x|^{1 / v}+|(x-b) / a|^{1 / v}\right\}} d x \\
& \leq \frac{C\left\|e^{h|x|^{1 / v}} f\right\|}{a^{1 / 2}} \int_{-\infty}^{\infty} e^{-\left(h-h^{\prime}\right)|x|^{1 / v}} d x \\
& \leq C\left\|e^{h|x|^{1 / v}} f\right\| .
\end{aligned}
$$


- Case of $0<v \leq 1$ and $0<a<1)$ Lemma 3.1 with $\alpha=|b-x|, \beta=|x|$ gives

$$
\begin{aligned}
& |x|^{1 / v}+\left|\frac{x-b}{a}\right|^{1 / v}-2^{1-1 / v}\left|\frac{b}{\max \{1, a\}}\right|^{1 / v} \\
& \quad=|b-x|^{1 / v}+|x|^{1 / v}-2^{1-1 / v}|b|^{1 / v}+|b-x|^{1 / v}\left\{\left|\frac{1}{a}\right|^{1 / v}-1\right\} \\
& \geq 2^{1-1 / v}(|b-x|+|x|)^{1 / v}-2^{1-1 / v}|b|^{1 / v}+|b-x|^{1 / v}\left\{\left|\frac{1}{a}\right|^{1 / v}-1\right\} \\
& \geq|b-x|^{1 / v}\left\{\left|\frac{1}{a}\right|^{1 / v}-1\right\} .
\end{aligned}
$$

Therefore, we have

$$
\begin{aligned}
\left|e^{h^{\prime} 2^{1-1 / v}|b / \max \{1, a\}|^{1 / v}} W_{\psi} f(a, b)\right| & \leq \frac{C\left\|e^{h|x|^{1 / v}} f\right\|}{a^{1 / 2}} \int_{-\infty}^{\infty} e^{-\left(h-h^{\prime}\right)\left\{|x|^{1 / v}+|(x-b) / a|^{1 / v}\right\}} d x \\
& \leq \frac{C\left\|e^{h|x|^{1 / v}} f\right\|}{a^{1 / 2}} \int_{-\infty}^{\infty} e^{-\left(h-h^{\prime}\right)|(x-b) / a|^{1 / v}} d x \\
& \leq C\left\|e^{h|x|^{1 / v}} f\right\| .
\end{aligned}
$$

Thus, since $\max \{1, a\} \leq a+1$, it follows that

$$
\left\|e^{h^{\prime} 2^{1-1 / v}|b /(a+1)|^{1 / v}} W_{\psi} f\right\| \leq C\left\|e^{h|x|^{1 / v}} f\right\|
$$

- Case of $\mu>1)$ Let $\mu^{\prime}:=\mu /(\mu+\delta)$. By Parseval's theorem, the wavelet transform can be rewritten as

$$
W_{\psi} f(a, b)=\sqrt{\frac{a}{C_{\psi}}} \int_{\mathbf{R}} \hat{f}(\xi) \overline{e^{-i b \xi} \hat{\psi}(a \xi)} d \xi .
$$

Since

$$
\left.e^{h \max \left\{|a \xi|^{1 / \mu},|a \xi|^{-1 / \delta}\right\}} \geq e^{h\left(|a \xi|^{1 / \mu}+|a \xi|^{-1 / \delta}-1\right)} \geq c e^{h\left(|a \xi|^{1 / \mu}+|a \xi|^{-1 / \delta}\right.}\right)
$$

similarly as (1), we see that

$$
\left.\left|e^{-i b \xi} \hat{\psi}(a \xi)\right| \leq C e^{-h\left(|a \xi|^{1 / \mu}+|a \xi|^{-1 / \delta}\right.}\right)
$$

Hence, we get

$$
\begin{aligned}
& \left|e^{h\left\{d(\delta / \mu+1)(1+1 / a)^{\mu^{\prime}}\right\}^{1 / \mu}} W_{\psi} f(a, b)\right| \\
& \quad \leq C a^{1 / 2}\left\|e^{h|\xi|^{1 / \mu}} \hat{f}\right\| \int_{-\infty}^{\infty} e^{-h\left\{|\xi|^{1 / \mu}+|a \xi|^{1 / \mu}+|a \xi|^{-1 / \delta}-\left\{d(\delta / \mu+1)(1+1 / a)^{\mu^{\prime}}\right\}^{1 / \mu}\right\}} d \xi .
\end{aligned}
$$

Lemma 3.1 with $\alpha=|\xi|, \beta=|a \xi|+|a \xi|^{-\mu / \delta}$ gives

$$
\begin{aligned}
& |\xi|^{1 / \mu}+|a \xi|^{1 / \mu}+\frac{1}{|a \xi|^{1 / \delta}}-\left\{d(\delta / \mu+1)\left(1+\frac{1}{a}\right)^{\mu^{\prime}}\right\}^{1 / \mu} \\
& \geq|\xi|^{1 / \mu}+\left(|a \xi|+\frac{1}{|a \xi|^{\mu / \delta}}\right)^{1 / \mu}-\left\{d(\delta / \mu+1)\left(1+\frac{1}{a}\right)^{\mu^{\prime}}\right\}^{1 / \mu}
\end{aligned}
$$




$$
\begin{aligned}
\geq & \left(|\xi|+|a \xi|+\frac{1}{|a \xi|^{\mu / \delta}}\right)^{1 / \mu}+\left(2-2^{1 / \mu}\right) \min \left\{|\xi|^{1 / \mu},\left(|a \xi|+\frac{1}{|a \xi|^{\mu / \delta}}\right)^{1 / \mu}\right\} \\
& -\left\{d(\delta / \mu+1)\left(1+\frac{1}{a}\right)^{\mu^{\prime}}\right\}^{1 / \mu} \\
\geq & \left(2-2^{1 / \mu}\right) \min \left\{|\xi|^{1 / \mu},\left(|a \xi|+\frac{1}{|a \xi|^{\mu / \delta}}\right)^{1 / \mu}\right\},
\end{aligned}
$$

here we used

$$
\min _{\xi \in \mathbf{R}}\left(|\xi|+|a \xi|+\frac{1}{|a \xi|^{\mu / \delta}}\right)=d\left(\frac{\delta}{\mu}+1\right)\left(1+\frac{1}{a}\right)^{\mu^{\prime}}
$$

Therefore, putting

$$
D:=\left\{\xi \in \mathbf{R} ;|\xi|^{1 / \mu}<\left(|a \xi|+|a \xi|^{-\mu / \delta}\right)^{1 / \mu}\right\}
$$

we have

$$
\begin{aligned}
\left|e^{h\left\{d(\delta / \mu+1)(1+1 / a)^{\mu^{\prime}}\right\}^{1 / \mu}} W_{\psi} f(a, b)\right| & \\
\leq & C a^{1 / 2}\left\|e^{h|\xi|^{1 / \mu}} \hat{f}\right\| \int_{D} e^{-h\left(2-2^{1 / \mu}\right)|\xi|^{1 / \mu}} d \xi \\
& +C a^{1 / 2}\left\|e^{h|\xi|^{1 / \mu}} \hat{f}\right\| \int_{\mathbf{R} \backslash D} e^{-h\left(2-2^{1 / \mu}\right)\left(|a \xi|+|a \xi|^{-\mu / \delta}\right)^{1 / \mu}} d \xi \\
\leq & \begin{cases}C a^{1 / 2}\left\|e^{h|\xi|^{1 / \mu}} \hat{f}\right\| \int_{\mathbf{R}} e^{-h\left(2-2^{1 / \mu}\right)|\xi|^{1 / \mu}} d \xi & \text { if } a \geq 1, \\
\frac{C\left\|e^{h|\xi|^{1 / \mu}} \hat{f}\right\|}{a^{1 / 2}} \int_{\mathbf{R}} e^{-h\left(2-2^{1 / \mu}\right)|\xi|^{1 / \mu}} d \xi & \text { if } 0<a<1\end{cases} \\
\leq & C \max \left\{a^{1 / 2}, a^{-1 / 2}\right\}\left\|e^{h|\xi|^{1 / \mu}} \hat{f}\right\| .
\end{aligned}
$$

Thus, since

$$
e^{h\left\{d(\delta / \mu+1)(1+1 / a)^{\mu^{\prime}}\right\}^{1 / \mu}} \geq e^{h\left\{d(\delta / \mu+1) a^{-\mu^{\prime}}\right\}^{1 / \mu}}=e^{h d(\delta / \mu+1)^{1 / \mu} a^{-1 /(\mu+\delta)}}
$$

and $\max \left\{a^{1 / 2}, a^{-1 / 2}\right\}=a^{-1 / 2} \max \{a, 1\} \sim a^{-1 / 2}(1+a)$, it follows that

$$
\left\|\frac{a^{1 / 2} e^{h d(\delta / \mu+1)^{1 / \mu} a^{-1 /(\mu+\delta)}}}{1+a} W_{\psi} f\right\| \leq C\left\|e^{h|\xi|^{1 / \mu}} \hat{f}\right\|
$$

- Case of $\mu>1$ with the condition $\left.|\hat{f}(\xi)| \leq C e^{-h|\xi|^{-1 / \delta}}\right)$ Let $\delta^{\prime}:=\delta /(\mu+\delta)$. By (2) we get

$$
\begin{aligned}
& \left|e^{h\left\{d(\delta / \mu+1)(1+a)^{\mu^{\prime}}\left(1+1 / a^{\mu / \delta}\right)^{\delta^{\prime}}\right\}^{1 / \mu}} W_{\psi} f(a, b)\right| \\
& \leq C a^{1 / 2}\left\|e^{h\left(\left.\xi\right|^{1 / \mu}+|\xi|^{-1 / \delta}\right)} \hat{f}\right\| \\
& \quad \times \int_{-\infty}^{\infty} e^{-h\left\{\left.\xi\right|^{1 / \mu}+|\xi|^{-1 / \delta}+|a \xi|^{1 / \mu}+|a \xi|^{-1 / \delta}-\left\{d(\delta / \mu+1)(1+a)^{\mu^{\prime}}\left(1+1 / a^{\mu / \delta}\right)^{\delta^{\prime}}\right\}^{1 / \mu}\right.} d \xi .
\end{aligned}
$$


Fukuda et al. Journal of Inequalities and Applications （2017) 2017:119

Page 10 of 24

Lemma 3.1 with $\alpha=|\xi|+|\xi|^{-\mu / \delta}, \beta=|a \xi|+|a \xi|^{-\mu / \delta}$ gives

$$
\begin{aligned}
|\xi|^{1 / \mu} & +\frac{1}{|\xi|^{1 / \delta}}+|a \xi|^{1 / \mu}+\frac{1}{|a \xi|^{1 / \delta}}-\left\{d(\delta / \mu+1)(1+a)^{\mu^{\prime}}\left(1+\frac{1}{a^{\mu / \delta}}\right)^{\delta^{\prime}}\right\}^{1 / \mu} \\
\geq & \left(|\xi|+\frac{1}{|\xi|^{\mu / \delta}}\right)^{1 / \mu}+\left(|a \xi|+\frac{1}{|a \xi|^{\mu / \delta}}\right)^{1 / \mu}-\left\{d(\delta / \mu+1)(1+a)^{\mu^{\prime}}\left(1+\frac{1}{a^{\mu / \delta}}\right)^{\delta^{\prime}}\right\}^{1 / \mu} \\
\geq & \left(|\xi|+\frac{1}{|\xi|^{\mu / \delta}}+|a \xi|+\frac{1}{|a \xi|^{\mu / \delta}}\right)^{1 / \mu} \\
& +\left(2-2^{1 / \mu}\right) \min \left\{\left(|\xi|+\frac{1}{|\xi|^{\mu / \delta}}\right)^{1 / \mu},\left(|a \xi|+\frac{1}{|a \xi|^{\mu / \delta}}\right)^{1 / \mu}\right\} \\
& \left.-\left\{d(\delta / \mu+1)(1+a)^{\mu^{\prime}}\left(1+\frac{1}{a^{\mu / \delta}}\right)^{\delta^{\prime}}\right\}^{1 / \mu}{ }^{1 / \mu}\left(|a \xi|+\frac{1}{|a \xi|^{\mu / \delta}}\right)^{1 / \mu}\right\} \\
\geq & \left(2-2^{1 / \mu}\right) \min \left\{\left(|\xi|+\frac{1}{|\xi|^{\mu / \delta}}\right)^{1 / \mu},(\mid a)\right.
\end{aligned}
$$

here we used

$$
\min _{\xi \in \mathbf{R}}\left(|\xi|+\frac{1}{|\xi|^{\mu / \delta}}+|a \xi|+\frac{1}{|a \xi|^{\mu / \delta}}\right)=d\left(\frac{\delta}{\mu}+1\right)(1+a)^{\mu^{\prime}}\left(1+\frac{1}{a^{\mu / \delta}}\right)^{\delta^{\prime}}
$$

Therefore, putting

$$
D:=\left\{\xi \in \mathbf{R} ;\left(|\xi|+|\xi|^{-\mu / \delta}\right)^{1 / \mu}<\left(|a \xi|+|a \xi|^{-\mu / \delta}\right)^{1 / \mu}\right\}
$$

we have

$$
\begin{aligned}
\left|e^{h\left\{d(\delta / \mu+1)(1+a)^{\mu^{\prime}}\left(1+1 / a^{\mu / \delta}\right)^{\delta^{\prime}}\right\}^{1 / \mu}} W_{\psi} f(a, b)\right| & \\
\leq & C a^{1 / 2}\left\|e^{h\left(|\xi|^{1 / \mu}+|\xi|^{-1 / \delta}\right)} \hat{f}\right\| \int_{D} e^{-h\left(2-2^{1 / \mu}\right)\left(|\xi|+|\xi|^{-\mu / \delta}\right)^{1 / \mu}} d \xi \\
& +C a^{1 / 2}\left\|e^{h\left(|\xi|^{1 / \mu}+|\xi|^{-1 / \delta}\right)} \hat{f}\right\| \int_{\mathbf{R} \backslash D} e^{-h\left(2-2^{1 / \mu}\right)\left(|a \xi|+|a \xi|^{-\mu / \delta}\right)^{1 / \mu}} d \xi \\
\leq & \begin{cases}C a^{1 / 2}\left\|e^{h\left(|\xi|^{1 / \mu}+|\xi|^{-1 / \delta}\right)} \hat{f}\right\| \int_{\mathbf{R}} e^{-h\left(2-2^{1 / \nu}\right)|\xi|^{1 / \mu}} d \xi & \text { if } a \geq 1, \\
\frac{C\left\|e^{h\left(\left.\xi\right|^{1 / \mu}+|\xi|^{-1 / \delta}\right)} \hat{f}\right\|}{a^{1 / 2}} \int_{\mathbf{R}} e^{-h\left(2-2^{1 / \nu}\right)|\xi|^{1 / \mu}} d \xi & \text { if } 0<a<1\end{cases} \\
\leq & C \max \left\{a^{1 / 2}, a^{-1 / 2}\right\}\left\|e^{h\left(|\xi|^{1 / \mu}+|\xi|^{-1 / \delta}\right)} \hat{f}\right\| .
\end{aligned}
$$

Thus, since

$$
(1+a)^{\mu^{\prime}}\left(1+\frac{1}{a^{\mu / \delta}}\right)^{\delta^{\prime}} \geq\left(\max \left\{a, \frac{1}{a}\right\}\right)^{\mu^{\prime}}
$$

and

$$
e^{h\left\{d(\delta / \mu+1)(1+a)^{\mu^{\prime}}\left(1+1 / a^{\mu / \delta}\right)^{\delta^{\prime}}\right\}^{1 / \mu}} \geq e^{h d(\delta / \mu+1)^{1 / \mu}\left(\max \left\{a, a^{-1}\right\}\right)^{1 /(\mu+\delta)}},
$$


it follows that

$$
\left\|\frac{a^{1 / 2} e^{h d(\delta / \mu+1)^{1 / \mu}\left(\max \left\{a, a^{-1}\right\}\right)^{1 /(\mu+\delta)}}}{1+a} W_{\psi} f\right\| \leq C\left\|e^{h\left(|\xi|^{1 / \mu}+\left.\xi\right|^{-1 / \delta}\right)} \hat{f}\right\| .
$$

- Case of $0<\mu \leq 1)$ Let $k=\max \{1 / \mu-1,1\}-1 / \delta$. For $h>h^{\prime}>0$, we get

$$
\begin{aligned}
& \left|e^{h^{\prime} 2^{1-1 / \mu}\left\{d(\delta / \mu+1)(1+1 / a)^{\mu^{\prime}}\right\}^{1 / \mu}} W_{\psi} f(a, b)\right| \\
& \leq C a^{1 / 2}\left\|e^{h|\xi|^{1 / \mu}} \hat{f}\right\| \\
& \times \int_{-\infty}^{\infty} e^{-\left(h-h^{\prime}\right)\left\{|\xi|^{1 / \mu}+|a \xi|^{1 / \mu}+|a \xi|^{-1 / \delta}\right\}} \\
& \times e^{-h^{\prime}\left\{|\xi|^{1 / \mu}+|a \xi|^{1 / \mu}+|a \xi|^{-1 / \delta}-2^{1-1 / \mu}\left\{d(\delta / \mu+1)(1+1 / a)^{\mu^{\prime}}\right\}^{1 / \mu}\right\}} d \xi .
\end{aligned}
$$

We note that if $\mu \neq 1$, i.e., $0<\mu<1$, there exists $L>0$ such that

$$
\begin{aligned}
\left(|a \xi|+\frac{1}{|a \xi|^{\mid \mu / \delta}}\right)^{1 / \mu} & =\frac{1}{|a \xi|^{1 / \delta}}\left(|a \xi|^{1+\mu / \delta}+1\right)^{1 / \mu} \\
& \leq \begin{cases}\frac{1}{|a \xi|^{1 / \delta}}\left(\left(|a \xi|^{1+\mu / \delta}\right)^{1 / \mu}+1^{1 / \mu}+L|a \xi|^{1 / \mu-1}\right) & \text { if }|a \xi| \geq 1, \\
\left.\frac{1}{|a \xi|^{1 / \delta}}\left(|a \xi|^{1+\mu / \delta}\right)^{1 / \mu}+1^{1 / \mu}+L|a \xi|\right) & \text { if } 0<|a \xi|<1\end{cases} \\
& \leq \frac{1}{|a \xi|^{1 / \delta}}\left(\left(|a \xi|^{1+\mu / \delta}\right)^{1 / \mu}+1^{1 / \mu}+L|a \xi|^{\max \{1 / \mu-1,1\}}\right) \\
& =|a \xi|^{1 / \mu}+\frac{1}{|a \xi|^{1 / \delta}}+L|a \xi|^{k} .
\end{aligned}
$$

If $\mu=1$, (4) also holds with $L=0$. Lemma 3.1 with $\alpha=|\xi|, \beta=|a \xi|+1 /|a \xi|^{\mu / \delta}$ gives

$$
\begin{aligned}
& |\xi|^{1 / \mu}+|a \xi|^{1 / \mu}+\frac{1}{|a \xi|^{1 / \delta}}-2^{1-1 / \mu}\left\{d(\delta / \mu+1)\left(1+\frac{1}{a}\right)^{\mu^{\prime}}\right\}^{1 / \mu} \\
& \quad \geq|\xi|^{1 / \mu}+\left(|a \xi|+\frac{1}{|a \xi|^{\mu / \delta}}\right)^{1 / \mu}-L|a \xi|^{k}-2^{1-1 / \mu}\left\{d(\delta / \mu+1)\left(1+\frac{1}{a}\right)^{\mu^{\prime}}\right\}^{1 / \mu} \\
& \quad \geq 2^{1-1 / \mu}\left(|\xi|+|a \xi|+\frac{1}{|a \xi|^{\mu / \delta}}\right)^{1 / \mu}-L|a \xi|^{k}-2^{1-1 / \mu}\left\{d(\delta / \mu+1)\left(1+\frac{1}{a}\right)^{\mu^{\prime}}\right\}^{1 / \mu} \\
& =-L|a \xi|^{k}
\end{aligned}
$$

here we used

$$
\min _{\xi \in \mathbf{R}}\left(|\xi|+|a \xi|+\frac{1}{|a \xi|^{\mu / \delta}}\right)=d(\delta / \mu+1)\left(1+\frac{1}{a}\right)^{\mu^{\prime}} .
$$

There exist $R \geq 1>r>0$ independent of $a>0$ such that

$$
L h^{\prime}|a \xi|^{k} \leq\left(h-h^{\prime}\right)\left(|a \xi|^{1 / \mu}+|a \xi|^{-1 / \delta}\right) \quad \text { for }|a \xi| \geq R \text { or }|a \xi|<r
$$

since $-1 / \delta<k=\max \{1 / \mu-1,1\}-1 / \delta<1 / \mu$. Therefore, putting

$$
I:=\{\xi \in \mathbf{R} ; r / a \leq|\xi|<R / a\},
$$


we have

$$
\begin{aligned}
&\left|e^{h^{\prime} 2^{1-1 / \mu}\left\{d(\delta / \mu+1)(1+1 / a)^{\mu^{\prime}}\right\}^{1 / \mu}} W_{\psi} f(a, b)\right| \\
& \leq C a^{1 / 2}\left\|e^{h|\xi|^{1 / \mu}} \hat{f}\right\| \int_{\mathbf{R}} e^{-\left(h-h^{\prime}\right)\left(|\xi|^{1 / \mu}+|a \xi|^{1 / \mu}+|a \xi|^{-1 / \delta}\right)+L h^{\prime}|a \xi|^{k}} d \xi \\
& \leq C a^{1 / 2}\left\|e^{h|\xi|^{1 / \mu}} \hat{f}\right\| \int_{\mathbf{R} \backslash I} e^{-\left(h-h^{\prime}\right)|\xi|^{1 / \mu}} d \xi \\
& \quad+C a^{1 / 2}\left\|e^{h|\xi|^{1 / \mu}} \hat{f}\right\| \int_{I} e^{-\left(h-h^{\prime}\right)\left(\left.\xi\right|^{1 / \mu}+|a \xi|^{1 / \mu}+|a \xi|^{-1 / \delta}\right)+L h^{\prime}|a \xi|^{k}} d \xi \\
& \leq C a^{1 / 2}\left\|e^{h|\xi|^{1 / \mu}} \hat{f}\right\| \int_{\mathbf{R}} e^{-\left(h-h^{\prime}\right)|\xi|^{1 / \mu}} d \xi \\
&+C a^{1 / 2}\left\|e^{h|\xi|^{1 / \mu}} \hat{f}\right\| \int_{\mathbf{R}} e^{-\left(h-h^{\prime}\right)|\xi|^{1 / \mu}+L h^{\prime} \max \left\{R^{k}, r^{k}\right\}} d \xi \\
& \leq C a^{1 / 2}\left\|e^{h|\xi|^{1 / \mu}} \hat{f}\right\| .
\end{aligned}
$$

Thus, it follows that

$$
\begin{aligned}
\left\|a^{-1 / 2} e^{h^{\prime} 2^{1-1 / \mu} d(\delta / \mu+1)^{1 / \mu} a^{-1 /(\mu+\delta)}} W_{\psi} f\right\| & \leq\left\|a^{-1 / 2} e^{h^{\prime} 2^{1-1 / \mu}\left\{d(\delta / \mu+1)(1+1 / a)^{\mu^{\prime}}\right\}^{1 / \mu}} W_{\psi} f\right\| \\
& \leq C\left\|e^{h|\xi|^{1 / \mu}} \hat{f}\right\| .
\end{aligned}
$$

- Case of $0<\mu \leq 1$ with the condition $|\hat{f}(\xi)| \leq C e^{-h|\xi|^{-1 / \delta}}$ ) For $h>h^{\prime}>0$, we get

$$
\begin{aligned}
& \left|e^{h^{\prime} 2^{1-1 / \mu}\left\{d(\delta / \mu+1)(1+a)^{\mu^{\prime}}\left(1+1 / a^{\mu / \delta}\right)^{\delta^{\prime}}\right\}^{1 / \mu}} W_{\psi} f(a, b)\right| \\
& \leq C a^{1 / 2}\left\|e^{h\left(|\xi|^{1 / \mu}+|\xi|^{-1 / \delta}\right)} \hat{f}\right\| \\
& \times \int_{-\infty}^{\infty} e^{-\left(h-h^{\prime}\right)\left\{|\xi|^{1 / \mu}+|\xi|^{-1 / \delta}+|a \xi|^{1 / \mu}+|a \xi|^{-1 / \delta}\right\}} \\
& \times e^{\left.-h^{\prime}|\xi|^{1 / \mu}+|\xi|^{-1 / \delta}+|a \xi|^{1 / \mu}+|a \xi|^{-1 / \delta}-2^{1-1 / \mu}\left\{d(\delta / \mu+1)(1+a)^{\mu^{\prime}}\left(1+1 / a^{\mu / \delta}\right)^{\delta^{\prime}}\right\}^{1 / \mu}\right\}} d \xi .
\end{aligned}
$$

By (4) Lemma 3.1 with $\alpha=|\xi|+1 /|\xi|^{\mu / \delta}, \beta=|a \xi|+1 /|a \xi|^{\mu / \delta}$ gives

$$
\begin{aligned}
&|\xi|^{1 / \mu}+|\xi|^{-1 / \delta}+|a \xi|^{1 / \mu}+\frac{1}{|a \xi|^{1 / \delta}}-2^{1-1 / \mu}\left\{d(\delta / \mu+1)(1+a)^{\mu^{\prime}}\left(1+\frac{1}{a^{\mu / \delta}}\right)^{\delta^{\prime}}\right\}^{1 / \mu} \\
& \geq\left(|\xi|+\frac{1}{|\xi|^{\mu / \delta}}\right)^{1 / \mu}+\left(|a \xi|+\frac{1}{|a \xi|^{\mu / \delta}}\right)^{1 / \mu}-L\left(|\xi|^{k}+|a \xi|^{k}\right) \\
&-2^{1-1 / \mu}\left\{d(\delta / \mu+1)(1+a)^{\mu^{\prime}}\left(1+\frac{1}{a^{\mu / \delta}}\right)^{\delta^{\prime}}\right\}^{1 / \mu} \\
& \geq 2^{1-1 / \mu}\left(|\xi|+\frac{1}{|\xi|^{\mu / \delta}}+|a \xi|+\frac{1}{|a \xi|^{\mu / \delta}}\right)^{1 / \mu}-L\left(|\xi|^{k}+|a \xi|^{k}\right) \\
&-2^{1-1 / \mu}\left\{d(\delta / \mu+1)(1+a)^{\mu^{\prime}}\left(1+\frac{1}{a^{\mu / \delta}}\right)^{\delta^{\prime}}\right\}^{1 / \mu} \\
&=-L\left(|\xi|^{k}+|a \xi|^{k}\right),
\end{aligned}
$$


here we used

$$
\min _{\xi \in \mathbf{R}}\left(|\xi|+\frac{1}{|\xi|^{\mu / \delta}}+|a \xi|+\frac{1}{|a \xi|^{\mu / \delta}}\right)=d(\delta / \mu+1)(1+a)^{\mu^{\prime}}\left(1+\frac{1}{a^{\mu / \delta}}\right)^{\delta^{\prime}} .
$$

There exist $R, \tilde{R} \geq 1>r, \tilde{r}>0$ independent of $a>0$ such that

$$
L h^{\prime}|a \xi|^{k} \leq\left(h-h^{\prime}\right)\left(|a \xi|^{1 / \mu}+|a \xi|^{-1 / \delta}\right) \quad \text { for }|a \xi|>R \text { or }|a \xi|<r,
$$

and for $\varepsilon>0$ satisfying $h>h^{\prime}+\varepsilon>h^{\prime}>0\left(\right.$ e.g., $\left.\varepsilon=\left(h-h^{\prime}\right) / 2\right)$

$$
L h^{\prime}|\xi|^{k} \leq\left(h-h^{\prime}-\varepsilon\right)\left(|\xi|^{1 / \mu}+|\xi|^{-1 / \delta}\right) \quad \text { for }|\xi|>\tilde{R} \text { or }|\xi|<\tilde{r}
$$

since $-1 / \delta<k=\max \{1 / \mu-1,1\}-1 / \delta<1 / \mu$. Therefore, putting

$$
I:=\{\xi \in \mathbf{R} ; r / a \leq|\xi|<R / a\} \text { and } J:=\{\xi \in \mathbf{R} ; \tilde{r} \leq|\xi|<\tilde{R}\}
$$

we have

$$
\begin{aligned}
& \left|e^{h^{\prime} 2^{1-1 / \mu}\left\{d(\delta / \mu+1)(1+a)^{\mu^{\prime}}\left(1+1 / a^{\mu / \delta}\right)^{\delta^{\prime}}\right\}^{1 / \mu}} W_{\psi} f(a, b)\right| \\
& \leq C a^{1 / 2}\left\|e^{h\left(|\xi|^{1 / \mu}+|\xi|^{-1 / \delta}\right)} \hat{f}\right\| \\
& \times \int_{\mathbf{R}} e^{-\left(h-h^{\prime}\right)\left(|\xi|^{1 / \mu}+|\xi|^{-1 / \delta}+|a \xi|^{1 / \mu}+|a \xi|^{-1 / \delta}\right)+L h^{\prime}\left(|\xi|^{k}+|a \xi|^{k}\right)} d \xi \\
& \leq C a^{1 / 2}\left\|e^{h\left(|\xi|^{1 / \mu}+|\xi|^{-1 / \delta}\right.} \hat{f}\right\|\left\{\int_{\mathbf{R} \backslash(I \cup)} e^{-\varepsilon\left(|\xi|^{1 / \mu}+|\xi|^{-1 / \delta}\right)} d \xi\right. \\
& +\int_{I \bigvee} e^{-\varepsilon\left(|\xi|^{1 / \mu}+|\xi|^{-1 / \delta}\right)-\left(h-h^{\prime}\right)\left(|a \xi|^{1 / \mu}+|a \xi|^{-1 / \delta}\right)+L h^{\prime}|a \xi|^{k}} d \xi \\
& +\int_{J \backslash I} e^{-\left(h-h^{\prime}\right)\left(|\xi|^{1 / \mu}+|\xi|^{-1 / \delta}\right)+L h^{\prime}|\xi|^{k}} d \xi \\
& \left.+\int_{I \cap J} e^{-\left(h-h^{\prime}\right)\left(|\xi|^{1 / \mu}+|\xi|^{-1 / \delta}+|a \xi|^{1 / \mu}+|a \xi|^{-1 / \delta}\right)+L h^{\prime}\left(|\xi|^{k}+|a \xi|^{k}\right)} d \xi\right\} \\
& \leq C a^{1 / 2}\left\|e^{h\left(|\xi|^{1 / \mu}+|\xi|^{-1 / \delta}\right)} \hat{f}\right\|\left\{\int_{\mathbf{R}} e^{-\varepsilon\left(|\xi|^{1 / \mu}+|\xi|^{-1 / \delta}\right)} d \xi\right. \\
& +\int_{\mathbf{R}} e^{-\varepsilon\left(|\xi|^{1 / \mu}+|\xi|^{-1 / \delta}\right)+L h^{\prime} \max \left\{R^{k}, r^{k}\right\}} d \xi \\
& +\int_{\mathbf{R}} e^{-\left(h-h^{\prime}\right)\left(\left.\xi \xi\right|^{1 / \mu}+|\xi|^{-1 / \delta}\right)+L h^{\prime} \max \left\{\tilde{R}^{k}, \tilde{r}^{k}\right\}} d \xi \\
& \left.+\int_{\mathbf{R}} e^{-\left(h-h^{\prime}\right)\left(|\xi|^{1 / \mu}+|\xi|^{-1 / \delta}\right)+L h^{\prime} \max \left\{R^{k}, \tilde{R}^{k}, r^{k}, \tilde{r}^{k}\right\}} d \xi\right\} \\
& \leq C a^{1 / 2}\left\|e^{h\left(|\xi|^{1 / \mu}+|\xi|^{-1 / \delta}\right)} \hat{f}\right\| \text {. }
\end{aligned}
$$

Thus, by (3) it follows that for $h>h^{\prime}>0$,

$$
\begin{aligned}
& \left\|a^{-1 / 2} e^{h 2^{1-1 / \mu} d(\delta / \mu+1)^{1 / \mu}\left(\max \left\{a, a^{-1}\right\}\right)^{1 /(\mu+\delta)}} W_{\psi} f\right\| \\
& \leq\left\|a^{-1 / 2} e^{h^{\prime} 2^{1-1 / \mu}\left\{d(\delta / \mu+1)(1+a)^{\mu^{\prime}}\left(1+1 / a^{\mu / \delta}\right)^{\delta^{\prime}}\right\}^{1 / \mu}} W_{\psi} f\right\| \leq C\left\|e^{h\left(|\xi|^{1 / \mu}+|\xi|^{-1 / \delta}\right)} \hat{f}\right\| .
\end{aligned}
$$

This concludes the proof of Theorem 2.4. 


\section{Concrete examples}

In this section, we introduce concrete examples according to whether the order of vanishing moments is finite or infinite.

- Case of finite vanishing moments) Let us consider the function and the wavelet

$$
f(x)=\operatorname{sech}(h x), \quad \psi(x)=\frac{d}{d x} \operatorname{sech}(h x) .
$$

In particular, when $h=\sqrt{\frac{\pi}{2}}$, it holds that $\hat{f}(\xi)=f(\xi)$, and we also see that $\hat{\psi}(\xi)=i \xi f(\xi)$ and $\psi \in S_{1, h^{\prime}}^{1, \infty}(\mathbf{R})$ with $0<h^{\prime}<h$. By the change of variables $t=e^{2 h a x}$, we have the wavelet transform

$$
\begin{aligned}
W_{\psi} f(a, b) & =\sqrt{\frac{a}{C_{\psi}}} \int_{\mathbf{R}} \psi(x) f(a x+b) d x \\
& =-4 h \sqrt{\frac{a}{C_{\psi}}} \int_{\mathbf{R}} \frac{e^{h x}-e^{-h x}}{\left(e^{h x}+e^{-h x}\right)^{2}} \cdot \frac{1}{e^{h(a x+b)}+e^{-h(a x+b)}} d x \\
& =-\frac{2}{e^{h b} \sqrt{C_{\psi} a}} \int_{0}^{\infty} \frac{t^{1 / a}-1}{\left(t^{1 / a}+1\right)^{2}} \cdot \frac{d t}{t^{\frac{a-1}{2 a}}\left(t+e^{-2 h b}\right)},
\end{aligned}
$$

where

$$
C_{\psi}=\int_{\mathbf{R}} \frac{|\hat{\psi}(\xi)|^{2}}{|\xi|} d \xi \quad(=2 \log 2) .
$$

Using the Hölder inequality $A+B \geq \frac{p}{(p-1)^{1-1 / p}} A^{1 / p} B^{1-1 / p}$ with

$$
1<\frac{2}{1+\frac{h^{\prime}}{h}}<p_{ \pm}:=\frac{2}{1 \pm \frac{h^{\prime}}{h \max \{1, a\}}}<\frac{2}{1-\frac{h^{\prime}}{h}}<\infty \quad \text { for } \operatorname{sgn} b= \pm 1 \text { respectively }
$$

we obtain the estimate (from above)

$$
\begin{aligned}
& \left|W_{\psi} f(a, b)\right| \\
& \leq \frac{2}{e^{h b} \sqrt{C_{\psi} a}} \int_{0}^{\infty} \frac{1}{t^{1 / a}+1} \cdot \frac{d t}{t^{\frac{a-1}{2 a}} \cdot \frac{p_{ \pm}}{\left(p_{ \pm}-1\right)^{1-1 / p_{ \pm}}} t^{1 / p_{ \pm}} e^{-2 h b\left(1-1 / p_{ \pm}\right)}} \\
& =\frac{2\left(p_{ \pm}-1\right)^{1-1 / p_{ \pm}} e^{h b\left(1-2 / p_{ \pm}\right)}}{p_{ \pm} \sqrt{C_{\psi} a}} \int_{0}^{\infty} \frac{1}{t^{1 / a}+1} \cdot \frac{d t}{t^{\frac{a-1}{2 a}+\frac{1}{p_{ \pm}}}} \\
& \leq \frac{C_{h^{\prime}} e^{h b\left(1-2 / p_{ \pm}\right)}}{\sqrt{a}} \int_{0}^{\infty} \frac{1}{t^{1 / a}+1} \cdot \frac{d t}{t^{1-\frac{1}{2 a} \pm} \frac{h^{\prime}}{2 h \max [1, a]}} \\
& \leq \frac{C_{h^{\prime}} e^{h b\left(1-2 / p_{ \pm}\right)}}{\sqrt{a}}\left\{\int_{0}^{1} \frac{1}{0+1} \cdot \frac{d t}{t^{1-\frac{1}{2 a} \pm \frac{h}{2 h \max (1, a)}}}+\int_{1}^{\infty} \frac{1}{t^{1 / a}+0} \cdot \frac{d t}{t^{1-\frac{1}{2 a} \pm \frac{h^{\prime}}{2 h \max \{1, a\}}}}\right\} \\
& =\frac{C_{h^{\prime}} e^{h b\left(1-2 / p_{ \pm}\right)}}{\sqrt{a}}\left\{\frac{1}{\frac{1}{2 a} \pm \frac{h^{\prime}}{2 h \max \{1, a\}}}+\frac{1}{\frac{1}{2 a} \mp \frac{h^{\prime}}{2 h \max \{1, a\}}}\right\} \\
& =\frac{C_{h^{\prime}} e^{h b\left(1-2 / p_{ \pm}\right)}}{\sqrt{a}} \frac{4 a}{1-\left(\frac{h^{\prime} a}{h \max \{1, a\}}\right)^{2}} \\
& \leq C_{h^{\prime}} a^{1 / 2} e^{-h^{\prime}|b| / \max \{1, a\}} \text {, }
\end{aligned}
$$


here we used the fact that

$$
1-\frac{1}{2 a} \pm \frac{h^{\prime}}{2 h \max \{1, a\}}<1, \quad 1+\frac{1}{2 a} \pm \frac{h^{\prime}}{2 h \max \{1, a\}}>1 .
$$

Then (i)' and (ii)' in Theorem 2.4 become

$$
\begin{aligned}
& \text { (i) })^{\prime} \quad\left\|e^{h^{\prime}|b /(a+1)|} W_{\psi} f\right\|_{L^{\infty}\left(\mathbf{R}_{+} \times \mathbf{R}\right)} \leq C\left\|e^{h|x|} f\right\|_{L^{\infty}(\mathbf{R})^{\prime}} \\
& \text { (ii) }^{\prime} \quad\left\|a^{-1 / 2} e^{h^{\prime}} W_{\psi} f\right\|_{L^{\infty}\left(\mathbf{R}_{+} \times \mathbf{R}\right)} \leq C\left\|e^{h|\xi| \hat{f}}\right\|_{L^{\infty}(\mathbf{R})} .
\end{aligned}
$$

From estimate (5) it is possible that this example is the near critical case of (i) ${ }^{\prime}$ and (ii) since $|b /(a+1)| \sim|b| / \max \{1, a\}$.

Remark 4.1 If we consider the typical example of the Mexican hat wavelet

$$
\psi(x)=\frac{2}{\pi^{1 / 4} \sqrt{3}}\left(1-x^{2}\right) e^{-x^{2} / 2}, \quad \hat{\psi}(\xi)=\frac{2 \sqrt{2 \pi}}{\pi^{1 / 4} \sqrt{3}} \xi^{2} e^{-\xi^{2} / 2}
$$

we see that $\psi \in S_{1 / 2, h^{\prime}}^{1 / 2, \infty}(\mathbf{R})$ with $0<h^{\prime}<h=1 / 2$. In particular, when $f(x)=e^{-x^{2} / 2} \in S_{1 / 2, h}^{1 / 2, \infty}(\mathbf{R})$, the wavelet transform is computed as

$$
W_{\psi} f(a, b)=\frac{2 \sqrt{2} \pi^{1 / 4} a^{5 / 2}\left(a^{2}-1-b^{2}\right)}{\sqrt{3 C_{\psi}}\left(a^{2}+1\right)^{5 / 2}} e^{-b^{2} /\left(2 a^{2}+2\right)} .
$$

Then (i)' in Theorem 2.4 becomes

$$
\text { (i) } \quad\left\|e^{h^{\prime} 2^{-1}|b /(a+1)|^{2}} W_{\psi} f\right\|_{L^{\infty}\left(\mathbf{R}_{+} \times \mathbf{R}\right)} \leq C\left\|e^{h|x|^{2}} f\right\|_{L^{\infty}(\mathbf{R})^{*}}
$$

The exponent $-b^{2} /\left(2 a^{2}+2\right)$ is not a critical case of (i) with $0<h^{\prime}<h=\frac{1}{2}$ since $h^{\prime} 2^{-1} \mid b /(a+$ 1) $\left.\right|^{2} \sim b^{2} /\left(4 a^{2}+4\right)$. Therefore, we gave the new wavelet $\psi(x)=\frac{d}{d x} \operatorname{sech}(h x) \in S_{1, h^{\prime}}^{1, \infty}(\mathbf{R})$ with $0<h^{\prime}<h=\sqrt{\frac{\pi}{2}}$.

- Case of infinite vanishing moments) Firstly we prove the following.

Proposition 4.2 The inverse Fourier transform of $e^{-\xi^{2}-t^{2} \xi^{-2}}$ is given by

$$
\mathcal{F}^{-1}\left[e^{-\xi^{2}-t^{2} \xi^{-2}}\right](x)=\frac{1}{\sqrt{2}} \sum_{n=0}^{\infty} \frac{(-2 t)^{n}}{n !}{ }_{1} F_{1}\left(\frac{1-n}{2}, \frac{1}{2},-\frac{x^{2}}{4}\right)
$$

where ${ }_{1} F_{1}(a, b, z)$ is the confluent hypergeometric function of the first kind.

Remark 4.3 The change of variables also yields

$$
\begin{aligned}
\mathcal{F}^{-1}\left[e^{\left.-\xi^{2} / 2-t^{2} \xi^{-2}\right](x)}\right. & =\sqrt{\frac{2}{\pi}} \int_{0}^{\infty} e^{-\xi^{2} / 2-t^{2} \xi^{-2}} \cos x \xi d \xi \\
& =\frac{2}{\sqrt{\pi}} \int_{0}^{\infty} e^{-\xi^{2}-(t / \sqrt{2})^{2} \xi^{-2}} \cos (\sqrt{2} x) \xi d \xi
\end{aligned}
$$




$$
\begin{aligned}
& =\sqrt{2} \mathcal{F}^{-1}\left[e^{-\xi^{2}-(t / \sqrt{2})^{2} \xi^{-2}}\right](\sqrt{2} x) \\
& =\sum_{n=0}^{\infty} \frac{(-\sqrt{2} t)^{n}}{n !}{ }_{1} F_{1}\left(\frac{1-n}{2}, \frac{1}{2},-\frac{x^{2}}{2}\right)
\end{aligned}
$$

Proof of Proposition 4.2 Let us put

$$
I(t, x):=\mathcal{F}^{-1}\left[e^{-\xi^{2}-t^{2} \xi^{-2}}\right](x)=\sqrt{\frac{2}{\pi}} \int_{0}^{\infty} e^{-\xi^{2}-t^{2} \xi^{-2}} \cos x \xi d \xi
$$

Differentiating $I(t, x)$ in $x$, we have

$$
\begin{aligned}
& \partial_{x} I(t, x)=-\sqrt{\frac{2}{\pi}} \int_{0}^{\infty} e^{-\xi^{2}-t^{2} \xi^{-2}} \xi \sin x \xi d \xi \\
& \partial_{x}^{2} I(t, x)=-\sqrt{\frac{2}{\pi}} \int_{0}^{\infty} e^{-\xi^{2}-t^{2} \xi^{-2}} \xi^{2} \cos x \xi d \xi
\end{aligned}
$$

On the other hand, differentiating $I$ in $t$, we also have

$$
\partial_{t} I(t, x)=-\sqrt{\frac{2}{\pi}} 2 t \int_{0}^{\infty} e^{-\xi^{2}-t^{2} \xi^{-2}} \xi^{-2} \cos x \xi d \xi
$$

Moreover, the integration by parts yields

$$
\begin{aligned}
\partial_{t} I(t, x) & =-\sqrt{\frac{2}{\pi}} t^{-1} \int_{0}^{\infty}\left\{e^{-t^{2} \xi^{-2}}\right\}^{\prime} e^{-\xi^{2}} \xi \cos x \xi d \xi \\
& =\sqrt{\frac{2}{\pi}} t^{-1} \int_{0}^{\infty} e^{-\xi^{2}-t^{2} \xi^{-2}}\left\{\left(1-2 \xi^{2}\right) \cos x \xi-x \xi \sin x \xi\right\} d \xi
\end{aligned}
$$

Thus, we see that $I(t, x)$ satisfies the partial differential equation

$$
\partial_{t} I(t, x)=2 t^{-1}\left\{\frac{1}{2} I(t, x)+\partial_{x}^{2} I(t, x)+\frac{x}{2} \partial_{x} I(t, x)\right\}
$$

We may suppose that $x \geq 0$ since $I(t, x)=\sqrt{\frac{2}{\pi}} \int_{0}^{\infty} e^{-\xi^{2}-t^{2} \xi^{-2}} \cos x \xi d \xi$ is an even function in $x$. Now we consider the point $x=2 \sqrt{-y}(y \leq 0)$ and get for $J(t, y):=I(t, 2 \sqrt{-y})$

$$
\partial_{y} J(t, y)=\frac{-1}{\sqrt{-y}}\left(\partial_{x} I\right)(t, 2 \sqrt{-y}), \quad \partial_{y}^{2} J(t, y)=-\frac{1}{y}\left(\partial_{x}^{2} I\right)(t, 2 \sqrt{-y})-\frac{1}{2 y} \partial_{y} J(t, y) .
$$

Therefore, by the change of variables $x=2 \sqrt{-y}$, it holds that

$$
\partial_{t} J(t, y)=2 t^{-1}\left\{\frac{1}{2} J(t, y)-y \partial_{y}^{2} J(t, y)+\left(y-\frac{1}{2}\right) \partial_{y} J(t, y)\right\} .
$$

To solve this partial differential equation, we shall use the method of separation of variables. By putting $J(t, y)=\sum_{n=0}^{\infty} L_{n}(t) K_{n}(y)$, we obtain

$$
\frac{t \partial_{t} L_{n}(t)}{2 L_{n}(t)}=\frac{-y \partial_{y}^{2} K_{n}(y)-\left(\frac{1}{2}-y\right) \partial_{y} K_{n}(y)+\frac{1}{2} K_{n}(y)}{K_{n}(y)}=: \lambda_{n}
$$


We immediately see that $L_{n}(t)=t^{2 \lambda_{n}} L_{n}(1)$. It is known that

$$
\begin{aligned}
I(t, 0) & =\sqrt{\frac{2}{\pi}} \int_{0}^{\infty} e^{-\xi^{2}-t^{2} \xi^{-2}} d \xi \\
& =\frac{1}{\sqrt{2}} e^{-2 t} \quad\left(\equiv \frac{1}{\sqrt{2}}\left\{1+\frac{(-2)}{1 !} t^{1}+\frac{(-2)^{2}}{2 !} t^{2}+\cdots\right\}\right) .
\end{aligned}
$$

We note that

$$
I(t, 0)=J(t, 0)=\sum_{n=0}^{\infty} L_{n}(t) K_{n}(0)=\sum_{n=0}^{\infty} L_{n}(t)
$$

here we may take $K_{n}(0)=1$ for all $n \in \mathbf{N}$ by choosing the suitable $L_{n}(t)$. Hence we see that $\lambda_{n}=\frac{n}{2}$ and

$$
L_{n}(t)=t^{n} L_{n}(1)=\frac{1}{\sqrt{2}} \frac{(-2)^{n}}{n !} t^{n}
$$

Meanwhile, the eigenvalue problem

$$
-y \partial_{y}^{2} K_{n}(y)-\left(\frac{1}{2}-y\right) \partial_{y} K_{n}(y)+\frac{1}{2} K_{n}(y)=\frac{n}{2} K_{n}(y)
$$

with $K_{n}(0)=1$ has

$$
K_{n}(y)={ }_{1} F_{1}\left(\frac{1-n}{2}, \frac{1}{2}, y\right)
$$

Thus it follows that

$$
J(t, y)=\sum_{n=0}^{\infty} L_{n}(t) K_{n}(y)=\sum_{n=0}^{\infty} \frac{1}{\sqrt{2}} \frac{(-2)^{n}}{n !} t^{n}{ }_{1} F_{1}\left(\frac{1-n}{2}, \frac{1}{2}, y\right),
$$

which gives

$$
I(t, x)=\frac{1}{\sqrt{2}} \sum_{n=0}^{\infty} t^{n} \frac{(-2)^{n}}{n !}{ }_{1} F_{1}\left(\frac{1-n}{2}, \frac{1}{2},-\frac{x^{2}}{4}\right) .
$$

We knew that $I(t, x)$ is an even function in advance and supposed that $x \geq 0$. The last representation also implies that $I(t, x)$ is an even function in $x$. So, (9) holds for all $x \in \mathbf{R}$.

We have derived (9) by solving the partial differential equation. To avoid confusion, let us denote the solution represented as in (9) by $\tilde{I}(t, x)$. It remains to show the uniqueness of $\tilde{I}(t, x)=\frac{1}{\sqrt{2}} \sum_{n=0}^{\infty} t^{n} \frac{(-2)^{n}}{n !}{ }_{1} F_{1}\left(\frac{1-n}{2}, \frac{1}{2},-\frac{x^{2}}{4}\right)$ and $I(t, x)=\sqrt{\frac{2}{\pi}} \int_{0}^{\infty} e^{-\xi^{2}-t^{2} \xi^{-2}} \cos x \xi d \xi$ except the case of $t=0$. Instead of $I(t, x)$, we consider for $(s, x) \in(0, \infty) \times \mathbf{R}$

$$
\mathcal{I}(s, x)(=I(\sqrt{s}, x))=\sqrt{\frac{2}{\pi}} \int_{0}^{\infty} e^{-\xi^{2}-s \xi^{-2}} \cos x \xi d \xi
$$


for the differentiation with respect to $s$. Then, by Stirling's formula, we obtain

$$
\begin{aligned}
\left|\partial_{s}^{m} \partial_{x}^{j} \mathcal{I}(s, x)\right| & \leq \sqrt{\frac{2}{\pi}} \int_{0}^{\infty} e^{-\xi^{2} / 2} \cdot e^{-s \xi^{-2}} \xi^{-2 m} \cdot e^{-\xi^{2} / 2}\left(\xi^{2}\right)^{j / 2} d \xi \\
& \leq C \sup _{\eta \geq 0} e^{-s \eta} \eta^{m} \cdot \sup _{\mu \geq 0} e^{-\mu / 2} \mu^{j / 2} \\
& \leq C e^{-m}\left(\frac{m}{s}\right)^{m} \cdot e^{-j / 2} j^{j / 2} \\
& \leq C r_{s}^{m+j} m !(j !)^{1 / 2} \quad\left(\leq C r_{s}^{m+j} m ! j !\right) .
\end{aligned}
$$

This implies that $\mathcal{I}(s, x)$ is analytic for $(s, x) \in\left[s_{0}, \infty\right) \times \mathbf{R}$ with arbitrarily fixed $s_{0}>0$. Therefore, we see that $I(t, x)=\sqrt{\frac{2}{\pi}} \int_{0}^{\infty} e^{-\xi^{2}-t^{2} \xi^{-2}} \cos x \xi d \xi$ is analytic for $(t, x) \in$ $(0, \infty) \times \mathbf{R}$.

Remark 4.4 Probably $I(t, x)$ would be analytic also at $t=0$. But $\mathcal{I}(s, x)(=I(\sqrt{s}, x))$ loses the analyticity at $s=0$. Indeed, we find that $I(\sqrt{s}, 0)=\frac{1}{\sqrt{2}} e^{-2 \sqrt{s}}=\frac{1}{\sqrt{2}}\left\{1+\frac{(-2)}{1 !} \sqrt{s}+\frac{(-2)^{2}}{2 !} s+\cdots\right\}$.

The Taylor expansion around a point $t=T>0$ gives

$$
I(t, x)\left(=\sqrt{\frac{2}{\pi}} \int_{0}^{\infty} e^{-\xi^{2}-t^{2} \xi^{-2}} \cos x \xi d \xi\right)=\sum_{n \geq 0, k \geq 0} a_{n, k}(t-T)^{n} x^{2 k}
$$

since $I(t, x)$ is an even function in $x$. By (9) we also get another Taylor expansion

$$
\tilde{I}(t, x)=\frac{1}{\sqrt{2}} \sum_{n=0}^{\infty}\{(t-T)+T\}^{n} \frac{(-2)^{n}}{n !}{ }_{1} F_{1}\left(\frac{1-n}{2}, \frac{1}{2},-\frac{x^{2}}{4}\right)=\sum_{n \geq 0, k \geq 0} \tilde{a}_{n, k}(t-T)^{n} x^{2 k} .
$$

Then $U(t, x):=I(t, x)-\tilde{I}(t, x)=\sum_{n \geq 0, k \geq 0} u_{n, k}(t-T)^{n} x^{2 k}$ satisfies

$$
\partial_{t} U(t, x)=2 t^{-1}\left\{\frac{1}{2} U(t, x)+\partial_{x}^{2} U(t, x)+\frac{x}{2} \partial_{x} U(t, x)\right\},
$$

and by (8)

$$
U(t, 0) \equiv 0
$$

Therefore, we get $u_{n, 0}=0$ for all $n \geq 0$ and

$$
\sum_{n \geq 1, k \geq 0} n u_{n, k} t(t-T)^{n-1} x^{2 k}=\sum_{n \geq 0, k \geq 0}(2 k+1)\left\{u_{n, k}+4(k+1) u_{n, k+1}\right\}(t-T)^{n} x^{2 k},
$$

here we used that

$$
\partial_{x}^{2} I=\sum_{n \geq 0, k \geq 1} 2 k(2 k-1) u_{n, k}(t-T)^{n} x^{2 k-2}=\sum_{n \geq 0, k \geq 0} 2(k+1)(2 k+1) u_{n, k+1}(t-T)^{n} x^{2 k} .
$$

Moreover, the left-hand side of (10) is changed into

$$
\sum_{n \geq 1, k \geq 0} n u_{n, k} t(t-T)^{n-1} x^{2 k}=\sum_{n \geq 0, k \geq 0}\left\{n u_{n, k}+(n+1) u_{n+1, k} T\right\}(t-T)^{n} x^{2 k} .
$$


Thus, it holds that

$$
n u_{n, k}+(n+1) u_{n+1, k} T=(2 k+1)\left\{u_{n, k}+4(k+1) u_{n, k+1}\right\} .
$$

Hence, when $u_{n, 0}=0$ for all $n \geq 0$, we find that $u_{n, 1}=0$ for all $n \geq 0$, and recursively $u_{n, k}=0$ for all $n \geq 0$ and $k \geq 0$. So, we have

$$
U(t, x)=\sum_{n \geq 0, k \geq 0} u_{n, k}(t-T)^{n} x^{2 k} \equiv 0
$$

This concludes that $I(t, x)\left(=\sqrt{\frac{2}{\pi}} \int_{0}^{\infty} e^{-\xi^{2}-t^{2} \xi^{-2}} \cos x \xi d \xi\right)$ must coincide with $\tilde{I}(t, x) \quad(=$ $\left.\frac{1}{\sqrt{2}} \sum_{n=0}^{\infty} t^{n} \frac{(-2)^{n}}{n !}{ }_{1} F_{1}\left(\frac{1-n}{2}, \frac{1}{2},-\frac{x^{2}}{4}\right)\right)$ for $(t, x) \in(0, \infty) \times \mathbf{R}$.

As an application of Proposition 4.2, we can compute the Fourier transform and the wavelet transform of concrete functions in the Gelfand-Shilov spaces. So, now let us take $\hat{\psi}(\xi)=\hat{f}(\xi)=e^{-\xi^{2}-\xi^{-2}}$. We see that $\psi, f \in S_{3 / 2, h}^{1 / 2,1 / 2}(\mathbf{R})$ for some $h>0$ since $e^{-\xi^{2}}$ gives $\mu=1 / 2$ and the Gevrey function $e^{-\xi^{-2}}$ gives $\delta=1 / 2$ and $v=3 / 2$ by the Paley-Wiener theorem. Then by (2) it follows that

$$
\begin{aligned}
W_{\psi} f(a, b) & =2 \sqrt{\frac{a}{C_{\psi}}} \int_{0}^{\infty} e^{-\left(1+a^{2}\right) \xi^{2}-\left(1+a^{-2}\right) \xi^{-2}} \cos b \xi d \xi \\
& =2 \sqrt{\frac{a}{C_{\psi}\left(1+a^{2}\right)}} \int_{0}^{\infty} e^{-\omega^{2}-\left(1+a^{-2}\right)\left(1+a^{2}\right) \omega^{-2}} \cos \frac{b}{\sqrt{1+a^{2}}} \omega d \omega \\
& =\sqrt{\frac{a}{C_{\psi}\left(1+a^{2}\right)}} \int_{-\infty}^{\infty} e^{-\omega^{2}-(a+1 / a)^{2} \omega^{-2}} e^{i \frac{b}{\sqrt{1+a^{2}}} \omega} d \omega \\
& =\sqrt{\frac{2 \pi a}{C_{\psi}\left(1+a^{2}\right)}} \mathcal{F}^{-1}\left[e^{-\omega^{2}-(a+1 / a)^{2} \omega^{-2}}\right]\left(\frac{b}{\sqrt{1+a^{2}}}\right) .
\end{aligned}
$$

By the Paley-Wiener theorem, we find that for some $\rho>0$

$$
\left|W_{\psi} f(a, b)\right| \leq C e^{-\rho\left|b / \sqrt{1+a^{2}}\right|^{2 / 3}} \sim C e^{-\rho|b /(1+a)|^{2 / 3}} .
$$

This implies that the order (i) in Theorem 2.4 is almost optimal with respect to $a$ and $b$. Using Proposition 4.2 with $t=1$ and $t=a+1 / a$, we have the following.

Theorem 4.5 Let $\hat{\psi}(\xi)=\hat{f}(\xi)=e^{-\xi^{2}-\xi^{-2}}$ for $\xi \neq 0$ and $=0$ for $\xi=0$. Then

$$
\psi(x)=f(x)=\frac{1}{\sqrt{2}} \sum_{n=0}^{\infty} \frac{(-2)^{n}}{n !}{ }_{1} F_{1}\left(\frac{1-n}{2}, \frac{1}{2},-\frac{x^{2}}{4}\right) \in S_{3 / 2, h}^{1 / 2,1 / 2}(\mathbf{R})
$$

for some $h>0$, and the wavelet transform is given by

$$
W_{\psi} f(a, b)=\sqrt{\frac{\pi a}{C_{\psi}\left(1+a^{2}\right)}} \sum_{n=0}^{\infty} \frac{\{-2(a+1 / a)\}^{n}}{n !}{ }_{1} F_{1}\left(\frac{1-n}{2}, \frac{1}{2},-\frac{b^{2}}{4\left(1+a^{2}\right)}\right),
$$

where ${ }_{1} F_{1}(a, b, z)$ is the confluent hypergeometric function of the first kind. 
Remark 4.6 Especially when $b=0$, we also find

$$
\left|W_{\psi} f(a, 0)\right|=\sqrt{\frac{\pi a}{C_{\psi}\left(1+a^{2}\right)}} e^{-2\left(a+\frac{1}{a}\right)} .
$$

Then (iii)' in Theorem 2.4 becomes

$$
\left\|a^{-1 / 2} e^{h^{\prime} 2 \max \left\{a, a^{-1}\right\}} W_{\psi} f\right\|_{L^{\infty}\left(\mathbf{R}_{+} \times \mathbf{R}\right)} \leq C\left\|e^{h \max \left\{|\xi|^{2},|\xi|^{-2}\right\}} \hat{f}\right\|_{L^{\infty}(\mathbf{R})} .
$$

(11) implies that $\max \left\{a, a^{-1}\right\}$ in (iii)' cannot be improved anymore since $h^{\prime} \sim 1$ and

$$
h^{\prime} 2 \max \left\{a, a^{-1}\right\} \sim 2\left(a+\frac{1}{a}\right) .
$$

Remark 4.7 As introduced in Remark 1.1, the Bessel wavelet $\psi(x)$ satisfies $\hat{\psi}(\xi)=e^{-\xi-\xi^{-1}}$ for $\xi>0$ and $\hat{\psi}(\xi)=0$ for $\xi \leq 0$ belongs to $\mathcal{S}_{2}^{1,+}(\mathbf{R})$. Hence, we also see that

$$
\psi(x)=\frac{1}{\pi \sqrt{1-i x}} K_{1}(2 \sqrt{1-i x})+\frac{1}{\pi \sqrt{1+i x}} K_{1}(2 \sqrt{1+i x})
$$

satisfies $\hat{\psi}(\xi)=e^{-|\xi|-|\xi|^{-1}}$ for $\xi \neq 0$ and $\hat{\psi}(\xi)=0$ for $\xi=0$ belongs to $\mathcal{S}_{2}^{1}(\mathbf{R})$ and $S_{2, h}^{1,1}(\mathbf{R})$ for some $h>0$.

\section{Conclusions}

In this paper, we consider the Banach spaces of Gelfand-Shilov functions satisfying vanishing moment conditions and study the wavelet transforms. Our contributions are as follows:

(1) We derived sharp estimates of the wavelet transforms which are useful for the time-frequency analysis, and stated the continuity properties of the wavelet transforms in Gelfand-Shilov spaces as a corollary.

(2) We computed the Fourier transforms and the wavelet transforms of concrete functions in the Gelfand-Shilov spaces. These examples show the optimality of estimates in Theorem 2.4.

\section{Appendix}

Concerned with the inverse wavelet transform, we also get the following.

Theorem A.1 Let $\mu, v, h, h^{\prime}$ and $\delta$ be positive constants such that $\mu+v \geq 1, h^{\prime}<h$. Define that $d(\lambda)=\lambda(\lambda-1)^{-1+1 / \lambda}$. Then, for the inverse wavelet transform $M_{\psi}$ with the wavelet $\psi \in S_{v, h}^{\mu, \delta}(\mathbf{R})$, the following estimates hold: for $F \in V_{v, h}^{\mu, \delta}\left(\mathbf{R}_{+} \times \mathbf{R}\right)$

(iv) $\left\|e^{h d(v / \mu+1)|x|^{1 /(\mu+\nu)}} M_{\psi} F\right\|_{L^{\infty}(\mathbf{R})}$

$$
\leq C\left\|e^{h\left\{|b / \max \{1, a\}|^{1 / v}+a^{1 / \mu}+a^{-1 / \delta}\right\}} F\right\|_{L^{\infty}\left(\mathbf{R}_{+} \times \mathbf{R}\right)} \text { if } v>1,
$$

(iv) $\quad\left\|e^{h^{\prime} 2^{1-1 / v} d(v / \mu+1)|x|^{1 /(\mu+\nu)}} M_{\psi} F\right\|_{L^{\infty}(\mathbf{R})}$

$$
\leq C\left\|e^{h\left\{|b / \max \{1, a\}|^{1 / v}+a^{1 / \mu}+a^{-1 / \delta}\right\}} F\right\|_{L^{\infty}\left(\mathbf{R}_{+} \times \mathbf{R}\right)} \quad \text { if } 0<\nu \leq 1,
$$


(v) $\left\|\frac{|\xi|^{1 / 2} e^{h d(\delta / \mu+1)^{1 / \mu}\left(\max \left\{|\xi|,|\xi|^{-1}\right\}\right)^{1 /(\mu+\delta)}}}{|\xi|+1} \mathcal{F}\left[M_{\psi} F\right]\right\|_{L^{\infty}(\mathbf{R})}$

$$
\leq\left\|e^{h\left\{|b / \max \{1, a\}|^{1 / v}+a^{1 / \mu}+a^{-1 / \delta}\right\}} F\right\|_{L^{\infty}\left(\mathbf{R}_{+} \times \mathbf{R}\right)} \quad \text { if } \mu>1,
$$

$(\mathrm{v})^{\prime} \quad\left\|e^{h^{\prime} 2^{1-1 / \mu}} d(\delta / \mu+1)^{1 / \mu}\left(\max \left\{|\xi|,|\xi|^{-1}\right\}\right)^{1 /(\mu+\delta)} \mathcal{F}\left[M_{\psi} F\right]\right\|_{L^{\infty}(\mathbf{R})}$

$$
\leq C\left\|e^{h\left\{|b / \max \{1, a\}|^{1 / v}+a^{1 / \mu}+a^{-1 / \delta}\right\}} F\right\|_{L^{\infty}\left(\mathbf{R}_{+} \times \mathbf{R}\right)} \quad \text { if } 0<\mu \leq 1 .
$$

The weight function of (iv) and (v) can be estimated as

$$
e^{h\left\{|b / \max \{1, a\}|^{1 / v}+a^{1 / \mu}+a^{-1 / \delta}\right\}} \leq e^{3 h \max \left\{|b /(a+1)|^{1 / v}, a^{1 / \mu}, a^{-1 / \delta}\right\}}
$$

and estimated from below as

$$
e^{h d(v / \mu+1)|x|^{1 /(\mu+v)}} \geq c e^{h|x|^{1 /(\mu+v)}}
$$

and

$$
\frac{|\xi|^{1 / 2} e^{h d(\delta / \mu+1)^{1 / \mu}\left(\max \left\{|\xi|,|\xi|^{-1}\right\}\right)^{1 /(\mu+\delta)}}}{|\xi|+1} \geq \frac{c e^{h\left\{|\xi|^{1 /(\mu+\delta)}+|\xi|^{-1 /(\mu+\delta)}\right\}}}{|\xi|^{1 / 2}+|\xi|^{-1 / 2}} \geq \frac{c}{2} e^{h\left\{|\xi|^{1 /(\mu+\delta)}+|\xi|^{-1 /(\mu+\delta)}\right\}}
$$

in the same way with (1). Therefore, by Theorem A.1, we can also get the following continuity property.

Corollary A.2 Let $\mu, v, h$ and $\delta$ be constants such that $\mu>1, v>1, h>0$ and $\delta>0$. Then, for the wavelet $\psi \in S_{v, h}^{\mu, \delta}(\mathbf{R})$, the inverse wavelet transform $V_{v, 3 h}^{\mu, \delta}\left(\mathbf{R}_{+} \times \mathbf{R}\right) \ni F \mapsto M_{\psi} F \in$ $S_{\mu+\nu, h}^{\mu+\delta, \mu+\delta}(\mathbf{R})$ is continuous.

We shall only give a sketch of the proof of Theorem A.1.

- Case of $v>1$ ) From the definition of the inverse wavelet transform we get

$$
\begin{aligned}
& \left|e^{h d(v / \mu+1)|x|^{1 /(\mu+v)}} M_{\psi} F(x)\right| \\
& \leq C\left\|e^{h\left\{|b / \max \{1, a\}|^{1 / v}+a^{1 / \mu}+a^{-1 / \delta}\right\}} F\right\| \\
& \quad \times \int_{\mathbf{R}_{+}} \int_{\mathbf{R}} a^{-5 / 2} e^{-h\left\{|b / \max \{1, a\}|^{1 / v}+a^{1 / \mu}+a^{-1 / \delta}+|(x-b) / a|^{1 / \nu}-d(v / \mu+1)|x|^{1 /(\mu+\nu)}\right\}} d b d a .
\end{aligned}
$$

We shall use the Hölder inequality $A+B \geq(A p)^{1 / p}(B q)^{1 / q}$ with $p=\mu / v+1, q=v / \mu+1$. If $a \geq 1$, Lemma 3.1 with $\alpha=|x / a-b / a|, \beta=|b / a|$ gives

$$
\begin{aligned}
& \left|\frac{b}{\max \{1, a\}}\right|^{1 / v}+a^{1 / \mu}+a^{-1 / \delta}+\left|\frac{x-b}{a}\right|^{1 / v}-d\left(\frac{v}{\mu}+1\right)|x|^{1 /(\mu+v)} \\
& \geq\left(2-2^{1 / v}\right) \min \left\{\left|\frac{x}{a}-\frac{b}{a}\right|^{1 / v},\left|\frac{b}{a}\right|^{1 / v}\right\}+\left|\frac{x}{a}\right|^{1 / v}+a^{1 / \mu}-d\left(\frac{v}{\mu}+1\right)|x|^{1 /(\mu+v)}+a^{-1 / \delta} \\
& \geq\left(2-2^{1 / v}\right) \min \left\{\left|\frac{x}{a}-\frac{b}{a}\right|^{1 / v},\left|\frac{b}{a}\right|^{1 / v}\right\}+a^{-1 / \delta} .
\end{aligned}
$$


If $0<a<1$, Lemma 3.1 with $\alpha=|x-b|, \beta=|b|$ gives

$$
\begin{aligned}
& \left|\frac{b}{\max \{1, a\}}\right|^{1 / v}+a^{1 / \mu}+a^{-1 / \delta}+\left|\frac{x-b}{a}\right|^{1 / v}-d\left(\frac{v}{\mu}+1\right)|x|^{1 /(\mu+v)} \\
& \geq|b|^{1 / v}+d\left(\frac{v}{\mu}+1\right)|x-b|^{1 /(\mu+v)}-d\left(\frac{v}{\mu}+1\right)|x|^{1 /(\mu+v)}+a^{-1 / \delta} \\
& \geq d\left(\frac{v}{\mu}+1\right)\left(2-2^{1 /(\mu+v)}\right) \min \left\{|x-b|^{1 /(\mu+v)},|b|^{1 /(\mu+v)}\right\}-m_{\mu, v}+a^{-1 / \delta}
\end{aligned}
$$

here we used

$$
|b|^{1 / v}-d\left(\frac{v}{\mu}+1\right)|b|^{1 /(\mu+v)} \geq-{ }^{\exists} m_{\mu, v} \quad \text { for } b \in \mathbf{R} .
$$

Thus, it follows that

$$
\begin{aligned}
\left\|e^{h d(v / \mu+1)|x|^{1 /(\mu+\nu)}} M_{\psi} F\right\| \leq & C\left\|e^{h\left\{|b / \max \{1, a\}|^{1 / v}+a^{1 / \mu}+a^{-1 / \delta}\right\}} F\right\| \\
& \times C\left\{\int_{1}^{\infty} a^{-3 / 2} e^{-h a^{-1 / \delta}} d a+\int_{0}^{1} a^{-5 / 2} e^{-h\left\{-m_{\mu, v}+a^{-1 / \delta}\right.} d a\right\} \\
\leq & C\left\|e^{h\left\{|b / \max \{1, a\}|^{1 / v}+a^{1 / \mu}+a^{-1 / \delta}\right\}} F\right\| .
\end{aligned}
$$

- Case of $0<v \leq 1$ ) This case can be shown similarly as the case of $v>1$.

- Case of $\mu>1$ ) This case can be shown similarly as the case of $\mu>1$ with the condition $|\hat{f}(\xi)| \leq C e^{-|\xi|^{1 / \delta}}$ for the wavelet transform by exchanging the roles of $a$ and $\xi$.

- Case of $0<\mu \leq 1$ ) For $h>h^{\prime}>0$, we get

$$
\begin{aligned}
& \left|e^{h^{\prime} 2^{1-1 / \mu}\left\{d(\delta / \mu+1)(1+|\xi|)^{\mu^{\prime}}\left(1+1 /|\xi|^{\mu / \delta} \delta^{\delta^{\prime}}\right\}^{1 / \mu}\right.} \mathcal{F}\left[M_{\psi} F\right](\xi)\right| \\
& \leq C\left\|e^{h\left\{|b / \max \{1, a\}|^{1 / \nu}+a^{1 / \mu}+a^{-1 / \delta}\right\}} F\right\| \int_{\mathbf{R}_{+}} \int_{\mathbf{R}} a^{-3 / 2} e^{-\left(h-h^{\prime}\right)\left\{a^{1 / \mu}+a^{-1 / \delta}+|a \xi|^{1 / \mu}+|a \xi|^{-1 / \delta}\right\}} \\
& \quad \times e^{-h^{\prime}\left\{|b / \max \{1, a\}|^{1 / v}+a^{1 / \mu}+a^{-1 / \delta}+|a \xi|^{1 / \mu}+|a \xi|^{-1 / \delta}-2^{1-1 / \mu}\left\{d(\delta / \mu+1)(1+|\xi|)^{\mu^{\prime}}\left(1+1 /|\xi|^{\mu / \delta}\right)^{\delta^{\prime}}\right\}^{1 / \mu}\right\}} d b d a .
\end{aligned}
$$

Similarly as the case of $0<\mu \leq 1$ for the wavelet transform, by (4) Lemma 3.1 with $\alpha=$ $a+a^{-\mu / \delta}, \beta=|a \xi|+|a \xi|^{-\mu / \delta}$ gives

$$
\begin{aligned}
& \left|\frac{b}{\max \{1, a\}}\right|^{1 / \nu}+a^{1 / \mu}+a^{-1 / \delta}+|a \xi|^{1 / \mu}+|a \xi|^{-1 / \delta} \\
& \quad-2^{1-1 / \mu}\left\{d\left(\frac{\delta}{\mu}+1\right)(1+|\xi|)^{\mu^{\prime}}\left(1+\frac{1}{|\xi|^{\mu / \delta}}\right)^{\delta^{\prime}}\right\}^{1 / \mu} \\
& \geq-L\left(a^{k}+|a \xi|^{k}\right)+\left|\frac{b}{\max \{1, a\}}\right|^{1 / v} .
\end{aligned}
$$

There exist $R, \tilde{R} \geq 1>r, \tilde{r}>0$ independent of $a>0$ such that

$$
L h^{\prime}|a \xi|^{k} \leq\left(h-h^{\prime}\right)\left(|a \xi|^{1 / \mu}+|a \xi|^{-1 / \delta}\right) \quad \text { for }|a \xi|>R \text { or }|a \xi|<r
$$


and for $\varepsilon>0$ satisfying $h>h^{\prime}+\varepsilon>h^{\prime}>0$ (e.g., $\left.\varepsilon=\left(h-h^{\prime}\right) / 2\right)$

$$
L h^{\prime} a^{k} \leq\left(h-h^{\prime}-\varepsilon\right)\left(a^{1 / \mu}+a^{-1 / \delta}\right) \quad \text { for } a>\tilde{R} \text { or } a<\tilde{r}
$$

since $-1 / \delta<k=\max \{1 / \mu-1,1\}-1 / \delta<1 / \mu$. Therefore, putting

$$
I:=\left\{a \in \mathbf{R}_{+} ; r /|\xi| \leq a<R /|\xi|\right\} \quad \text { and } \quad J:=\left\{a \in \mathbf{R}_{+} ; \tilde{r} \leq a<\tilde{R}\right\}
$$

we have

$$
\begin{aligned}
&\left|e^{h^{\prime} 2^{1-1 / \mu}\left\{d(\delta / \mu+1)(1+|\xi|)^{\mu^{\prime}}\left(1+1 /|\xi|^{\mu / \delta}\right)^{\delta^{\prime}}\right\}^{1 / \mu}} \mathcal{F}\left[M_{\psi} F\right](\xi)\right| \\
& \leq C\left\|e^{h\left\{|b / \max \{1, a\}|^{1 / v}+a^{1 / \mu}+a^{-1 / \delta}\right\}} F\right\| \\
& \times \int_{\mathbf{R}_{+}} \min \left\{a^{-1 / 2}, a^{-3 / 2}\right\} e^{-\left(h-h^{\prime}\right)\left\{a^{1 / \mu}+a^{-1 / \delta}+|a \xi|^{1 / \mu}+|a \xi|^{-1 / \delta}\right\}+L h^{\prime}\left(a^{k}+|a \xi|^{k}\right)} d a \\
& \leq C\left\|e^{h\left\{|b / \max \{1, a\}|^{1 / v}+a^{1 / \mu}+a^{-1 / \delta}\right\}} F\right\|\left\{\int_{\mathbf{R}_{+} \backslash(I \cup J)} \min \left\{a^{-1 / 2}, a^{-3 / 2}\right\} e^{-\varepsilon\left(a^{1 / \mu}+a^{-1 / \delta}\right)} d a\right. \\
&+\int_{I V} \min \left\{a^{-1 / 2}, a^{-3 / 2}\right\} e^{-\varepsilon\left(a^{1 / \mu}+a^{-1 / \delta}\right)-\left(h-h^{\prime}\right)\left(|a \xi|^{1 / \mu}+|a \xi|^{-1 / \delta}\right)+L h^{\prime}|a \xi|^{k}} d a \\
&+\int_{J \backslash I} \min \left\{a^{-1 / 2}, a^{-3 / 2}\right\} e^{-\left(h-h^{\prime}\right)\left(a^{1 / \mu}+a^{-1 / \delta}\right)+L h^{\prime} a^{k}} d a \\
&\left.+\int_{I \cap J} \min \left\{a^{-1 / 2}, a^{-3 / 2}\right\} e^{-\left(h-h^{\prime}\right)\left(a^{1 / \mu}+a^{-1 / \delta}+|a \xi|^{1 / \mu}+|a \xi|^{-1 / \delta}\right)+L h^{\prime}\left(a^{k}+|a \xi|^{k}\right)} d a\right\} \\
& \leq C\left\|e^{h\left\{|b / \max \{1, a\}|^{1 / v}+a^{1 / \mu}+a^{-1 / \delta}\right\}} F\right\| .
\end{aligned}
$$

Thus, by the inequality $(1+|\xi|)^{\mu^{\prime}}\left(1+\frac{1}{|\xi|^{\mid / \delta}} \delta^{\delta^{\prime}} \geq\left(\max \left\{|\xi|, \frac{1}{|\xi|}\right\}\right)^{\mu^{\prime}}\right.$, it follows that

$$
\left\|e^{h^{\prime} 2^{1-1 / \mu}} d(\delta / \mu+1)^{1 / \mu}\left(\max \left\{|\xi|,|\xi|^{-1}\right\}\right)^{1 / \mu+\delta)} \mathcal{F}\left[M_{\psi} F\right]\right\| \leq\left\|e^{h\left\{|b / \max \{1, a\}|^{1 / v}+a^{1 / \mu}+a^{-1 / \delta}\right\}} F\right\| .
$$

\section{Competing interests}

The authors declare that they have no competing interests.

\section{Authors' contributions}

This work was carried out in collaboration among all authors. The author TK plays the role of corresponding author. All authors read and approved the final manuscript.

\section{Author details}

${ }^{1}$ Matsue College, National Institute of Technology, Matsue, Shimane 690-8518, Japan. ${ }^{2}$ Institute of Mathematics, Tsukuba University, Tsukuba, Ibaraki 305-8571, Japan.

\section{Acknowledgements}

This work was supported by Grant-in-Aid for Scientific Research (C) (No. 16K05223), Japan Society for the Promotion of Science. The authors appreciate the reviewers for their constructive comments to improve the quality of the paper. The authors also wish to thank Prof. Kunio Yoshino for valuable suggestions.

\section{Publisher's Note}

Springer Nature remains neutral with regard to jurisdictional claims in published maps and institutional affiliations. 
References

1. Daubechies, I: Ten Lectures on Wavelets. CBMS-NSF Regional Conference Series in Applied Mathematics, vol. 61. SIAM, Philadelphia (1992)

2. Mallat, S: A Wavelet Tour of Signal Processing, 3rd edn. The Sparse Way. Elsevier, Amsterdam (2009)

3. Pathak, RS: The wavelet transform of distributions. Tohoku Math. J. (2) 56(3), 411-421 (2004)

4. Gelfand, IM, Shilov, GE: Generalized Functions, vol. 2. Academic Press, New York (1968)

5. Gelfand, IM, Shilov, GE: Generalized Functions, vol. 3. Academic Press, New York (1967)

6. Nicola, F, Rodino, L: Global Pseudo-Differential Calculus on Euclidean Spaces. Pseudo-Differential Operators, Theory and Applications, vol. 4. Birkhäuser, Basel (2010)

7. Pilipović, S, Rakić, D, Teofanov, N, Vindas, J: The wavelet transforms in Gelfand-Shilov spaces. Collect. Math. 67(3), 443-460 (2016)

8. Grossmann, A, Morlet, J: Decomposition of Hardy functions into square integrable wavelets of constant shape. SIAM J. Math. Anal. 15(4), 723-736 (1984)

9. Holschneider, M: Wavelets. An Analysis Tool. Oxford Mathematical Monographs. Oxford Science Publications. Clarendon, New York (1995)

10. Fukuda, N, Kinoshita, T, Uehara, I: On the wavelets having Gevrey regularities and subexponential decays. Math. Nachr. 287, 546-560 (2014)

11. Rakić, D, Teofanov, N: Progressive Gelfand-Shilov spaces and wavelet transforms. J. Funct. Spaces Appl. 2012, Article ID $951819(2012)$

12. Chung, J, Chung, SY, Kim, D: Characterizations of the Gelfand-Shilov spaces via Fourier transforms. Proc. Am. Math. Soc. 124, 2101-2108 (1996)

13. Bourdaud, G: Sur les opérateurs pseudo-différentiels à coefficients peu réguliers. Thesis, Univ. Paris-Sud, Paris (1983)

14. Reich, M, Reissig, M, Sickel, W: Non-analytic superposition results on modulation spaces with subexponential weights. J. Pseud.-Differ. Oper. Appl. 7(3), 36-409 (2016)

\section{Submit your manuscript to a SpringerOpen ${ }^{\circ}$ journal and benefit from:}

- Convenient online submission

- Rigorous peer review

Immediate publication on acceptance

Open access: articles freely available online

- High visibility within the field

- Retaining the copyright to your article 